\title{
A Multi-Component Analysis Indicates a Positronic Major Flare in GRS 1915+105
}

\author{
Brian Punsly ${ }^{1}$
}

\begin{abstract}
A modeling strategy that is adapted to the study of synchrotron-self absorbed plasmoids that was developed for the quasar, Mrk 231, in Reynolds et al (2009) is applied to the microquasar GRS 1915+105. The major flare from December 1993 shows spectral evidence of three such self-absorbed components. The analysis yields an estimate of the power that is required to eject the plasmoids from the central engine that is independent of other estimates that exist in the literature for different flares. The technique has an advantage since the absorbed spectrum contains an independent constraint provided by the optical depth at each epoch of observation. The modeling procedure presented here self-consistently determines the dimensions of the radio emitting plasma from the spectral shape. Thus, structural dimensions are determined analytically that can be much smaller than interferometer beam-widths. A synthesis of the time evolution of the components allows one to address the fundamental uncertainties in previous estimates. First, the plasma is not protonic, but it is comprised of an electron-positron gas. The minimum electron energy is determined to be less than six times the electron rest mass energy. The analysis also indicates that the plasmoids are ejected from the central engine magnetically dominated. The temporal behavior is one of magnetic energy conversion to mechanical energy as the plasmoids approach equipartition. The time dependent models bound the impulsive energy flux, $Q$, required to eject the individual major flare plasmoids from the central engine to, $4.1 \times 10^{37} \mathrm{erg} / \mathrm{s}<Q<6.1 \times 10^{38} \mathrm{ergs} / \mathrm{s}$.
\end{abstract}

Subject headings: Black hole physics — X-rays: binaries — accretion, accretion disks

\footnotetext{
${ }^{1} 1415$ Granvia Altamira, Palos Verdes Estates, CA USA 90274 and ICRANet, Piazza della Repubblica 10 Pescara 65100, Italy, brian.punsly@verizon.net or brian.punsly@comdev-usa.com
} 


\section{Introduction}

It is possible that Galactic black hole accretion systems can serve as laboratories in which AGN (active galactic nuclei) behavior can be observed on time scales that are highly compressed. The black hole candidate, GRS $1915+105$ is of particular interest because of a possible analogy with radio loud AGN. It routinely ejects powerful radio emitting plasma out to large distances from the black hole at relativistic speeds (Mirabel and Rodriguez 1994; Rodriguez and Mirabel 1999; Fender et al 1999; Miller-Jones et al 2005). There is no other Galactic black hole that has produced nearly as many strong radio flares that have been observed to be superluminal. The propensity for high radio flux densities and superluminal ejections make this Galactic black hole ideal for studying relativistic jet formation. The superluminal ejections (jet) has been resolved into multiple components at late times with each component limited in size by the interferometer beamwidth (Fender et al 1999; Dhawan et al 2000). It is also noteworthy that many of these states of high radio activity are compact op-

tically thick jets in which the X-ray spectrum is hard (Dhawan et al 2000; Ribo et al 2004). The X-ray luminosity of GRS $1915+105$ is also one of the highest of any known Galactic black hole candidate (Done et al 2004). Unlike other black holes in which the compact jet occurs in a "low hard" spectral state, the X-ray luminosity of the GRS 1915+105 can be very high $(\approx 20 \%$ of the Eddington limit) in the hard state when a compact jet is present (Fuchs et al 2003). It is not clear if the the two remarkable properties of a luminous accretion flow and extraordinary superluminal ejections are related. The existence of both properties make it tempting to speculate that GRS $1915+105$ is a scaled down version of a radio loud quasar.

In order to understand the temporal relationship between the episodic energy ejection mechanism and the accretion state, one must be able to establish the magnitude of the mechanical energy flux in these ejecta. The major flare in 1994 is well chronicled (Mirabel and Rodriguez 1994). Unfortunately, minimum energy estimates of the kinetic luminosity, $Q$, vary by orders of magnitude $10^{37} \mathrm{erg} / \mathrm{s}<Q<7 \times 10^{41} \mathrm{ergs} / \mathrm{s}$ (Gliozzi et al 1999; Rodriguez et al 1995; Liang and Li 1995; Fender et al 1999). This paper uses techniques developed in Reynolds et al (2009) to constrain these estimates and remove the layers of assumptions. Previous efforts to estimate jet power have been plagued by various unknowns. Each one of these unknowns can independently contribute an order of magnitude or more of uncertainty to the estimates. The four major ones are:

1. Is the plasma protonic? It is often assumed that the plasmoid is either protonic (normal electron - proton gas) or positronic (a pair plasma). Proton kinetic energy will typically dominate in a relativistic outflow. Major flares have optically thin radio spectra at late times. The energy of the synchrotron emitting leptons is then dominated by low energy 
particles and the particle number density becomes high. If the radio spectrum extends to low frequency and neutrality is maintained by protons, the energy flux in the protons can be more than two orders of magnitude larger than the leptonic energy flux.

2. What is the minimum electron energy, $E_{\min }$ ? Again, this is given by fiat in previous estimates. If $E_{\text {min }}=1$ in units of the electron rest mass energy, $m_{e} c^{2}$, then the radio spectrum will extend to frequencies below those observed and the total number of particles increases dramatically for optically thin radio emitting plasmoids. The value of $E_{\text {min }}$ is another parameter in which orders of magnitude of energy uncertainty is hidden.

3. There is uncertainty in the size of the region that produces the bulk of the radio emission. The kinetic luminosity of the plasma, $Q$, that produces the radio emission scales with the size of the emitting region. The size of the physical region that produces the preponderance of the radio emission in major flares appears to be smaller than the FWHM (full width at half maximum) beamwidth of the VLA, VLBA or MERLIN interferometers as evidenced by the VLBA and MERLIN images in Rodriguez and Mirabel (1999); Dhawan et al (2000); Fender et al (1999). The existence of unresolved components, significantly smaller than the beamwidth FWHM, would indicate that the size of the emitting region has likely been typically over-estimated in the past. Since the interferometer beamwidth is potentially much larger than the plasmoid size, extremely liberal sizes have been chosen a priori for energy estimates. Examples that are mentioned in the Discussion section are:

- the entire deconvolved angular dimensions of an unresolved "jet" that was partially resolved from an adjacent radio component has been used as the size of the radio emitting plasmoid projected onto the sky plane in Gliozzi et al (1999); Rodriguez and Mirabel (1999)

- and an assumed speed of light expansion of the plasmoid from an estimated initiation time was used as the plasmoid size in Fender et al (1999).

These could be considered as the most extreme upper bounds on the plasmoid size that are compatible with a subset of the data rather than estimates for the plasmoid size. For a minimum energy estimate of a spherical emission region of radius, $r, Q \sim r^{9 / 7}$ (Liang and Li 1995; Fender et al 1999). Thus, order of magnitude errors in the size of the region of the preponderance of emissivity result in even larger errors in the estimates for $Q$.

4. Is the minimum energy or the equipartition assumption justified? Such an assumption is typically made just to obtain an extra constraint that can be used to restrict the 
variables in the calculation of the energy flux. Yet, there is no evidence that this assumption is justified.

In Reynolds et al (2009), a powerful modeling method for analyzing relativistic plasma ejections was found in the context of the quasar, Mrk 231. The modeling method exploits the fact that synchrotron self-absorbed plasmoids are restricted physically by the shape of their spectrum. In particular, the frequency and the width of the spectral peak. This provides two added pieces of information beyond the spectral index and flux density of the optically thin high frequency tail (i.e., the only information that is used in other estimates of the jet power of GRS 1915+105). Such an analysis provides strong constraints on the size of the emitting region. In order to implement the modeling method, one must have sufficient frequency coverage so that the peak and the high frequency tail of the spectrum are defined. Furthermore, the data must be quasi-simultaneous because strong flares evolve rapidly, especially in their compact self-absorbed phase. A literature search revealed one such instance of broadband simultaneous frequency coverage in Rodriguez et al (1995) for a flare in December 1993. The modeling of the self-absorbed components of this flare and their time evolution is the subject of this study. The next section describes the synchrotron self-absorbed fits to the spectra. Section 3 provides the range of physical parameters that are consistent with these fits. Section 4 discusses the possibility of protonic plasma in the synchrotron emitting region. Section 5 uses the time evolution information to bound the power required to create the relativistic ejecta.

\section{The Major Flare of December 1993}

The radio monitoring of Rodriguez et al (1995) revealed that a large flare had emerged from GRS 1915+105 on December 6, 1993. The flare was of comparable in magnitude to the large flare of March 1994 described in Mirabel and Rodriguez (1994). The December 1993 flare was unique in that there was spectral coverage all the way from $1.465 \mathrm{GHz}$ to $234 \mathrm{GHz}$. The data from Rodriguez et al (1995) is repeated in Table 1. There are three dates that are well sampled.There are 7 data points for December 6, 4 data points for December 11, and 8 data points for December 14 for a total of 19 data points. This a bit deceiving because 3 data points on December 14 are all at $\approx 1.4 \mathrm{GHz}$. Thus there are really only 6 independent data points on December 14, for a total of 17 independent data points on the three days. Notice that all 3 of the $1.4 \mathrm{GHz}$ observations on December 14 are the same within the quoted errors. This indicates that the optically thin spectrum was relatively stable over time scales of at least 4.5 hours.

In this section, fits to the data in Table 1 are presented using synchrotron self absorbed 
Two Component Fit to Simultaneous VLA Data on 12/6/1993

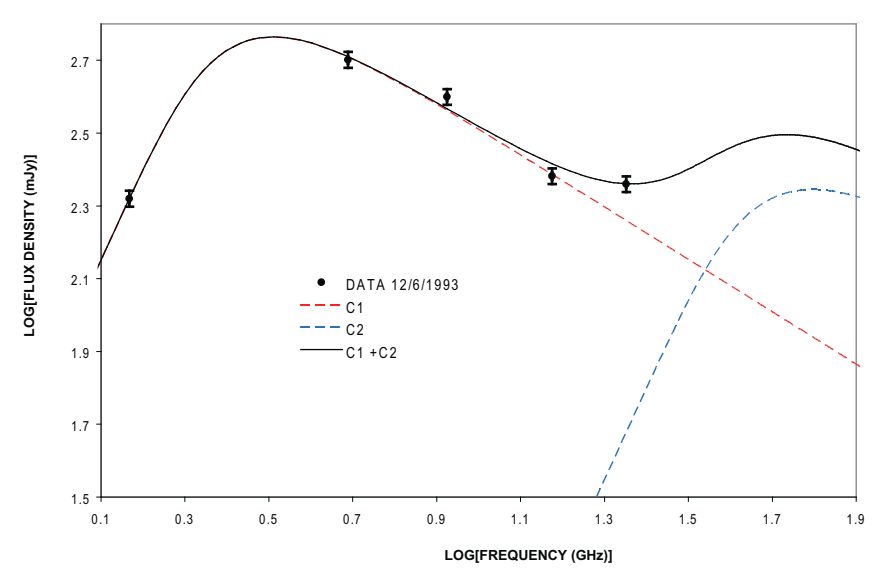

Three Component Fit to Simultaneous VLA Data on 12/6/1993

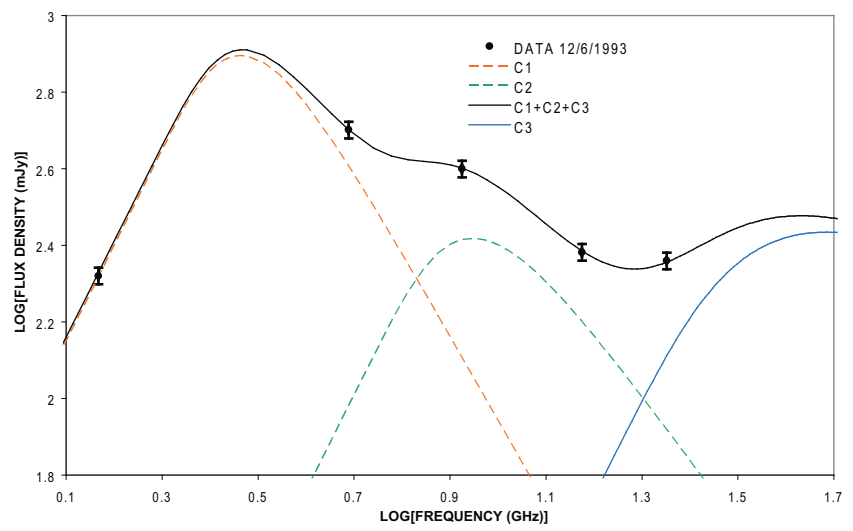

Fig. 1. - A comparison of a two component fit and a three component fit of the simultaneous VLA data on December 6. 


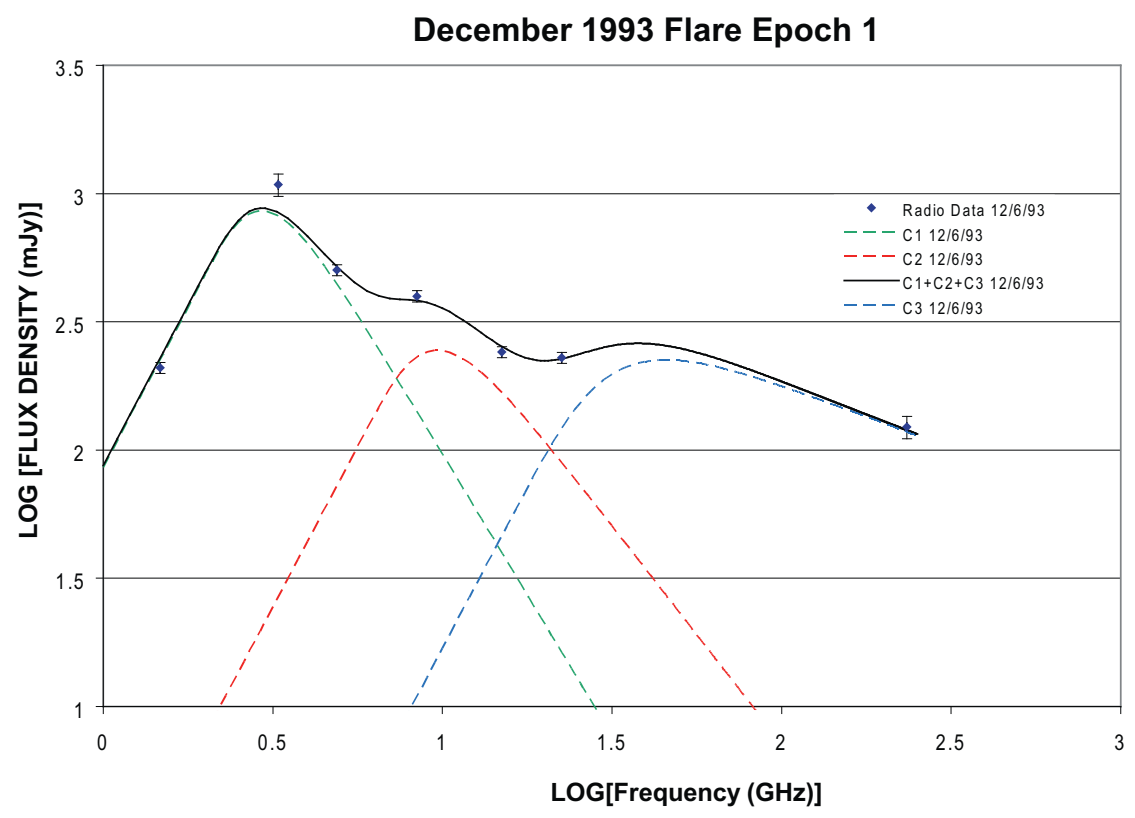

Fig. 2. - The broad band quasi-simultaneous spectrum of GRS 1915+105 on December 6 , 1993. The data is fit to three synchrotron self-absorbed components. They are labeled $\mathrm{C} 1, \mathrm{C} 2$ and $\mathrm{C} 3$. The component $\mathrm{C} 1$ is the lowest frequency peaked component and $\mathrm{C} 3$ the highest. The $3.277 \mathrm{GHz}$ data and the $234 \mathrm{GHz}$ data is taken 12 hours later than the other data on the plot. 


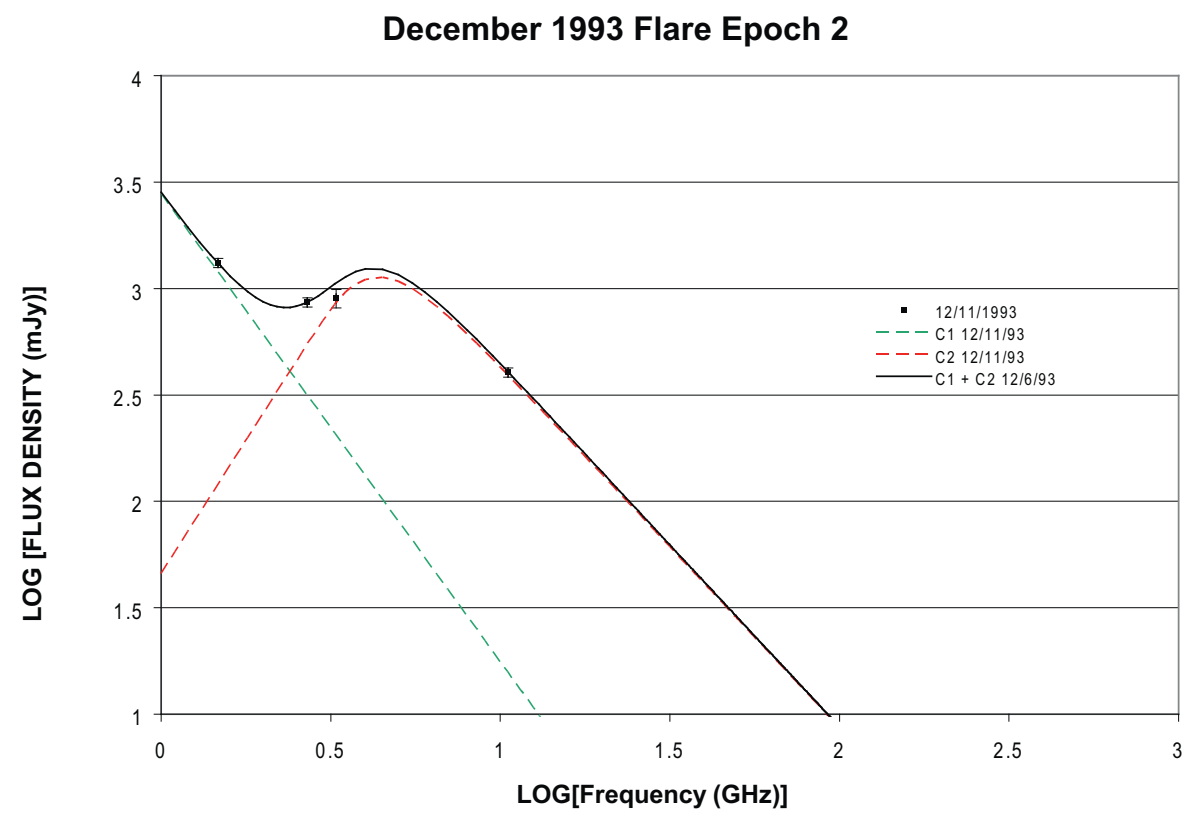

Fig. 3.- The spectrum of GRS 1915+105 on December 11, 1993. The frequency coverage is certainly more sparse than on December 6 . But, there is enough data to constrain a local maximum near $5 \mathrm{GHz}$ that is attributed to component $\mathrm{C} 2$. Since the frequency coverage ends at $10.5 \mathrm{GHz}$, there is an insignificant contribution expected from the high frequency component, $\mathrm{C} 3$, to this data. Both $\mathrm{C} 1$ and $\mathrm{C} 2$ are assumed to have the same value of $\alpha$ as for epoch December 6, 1993 
December 1993 Flare Epoch 3

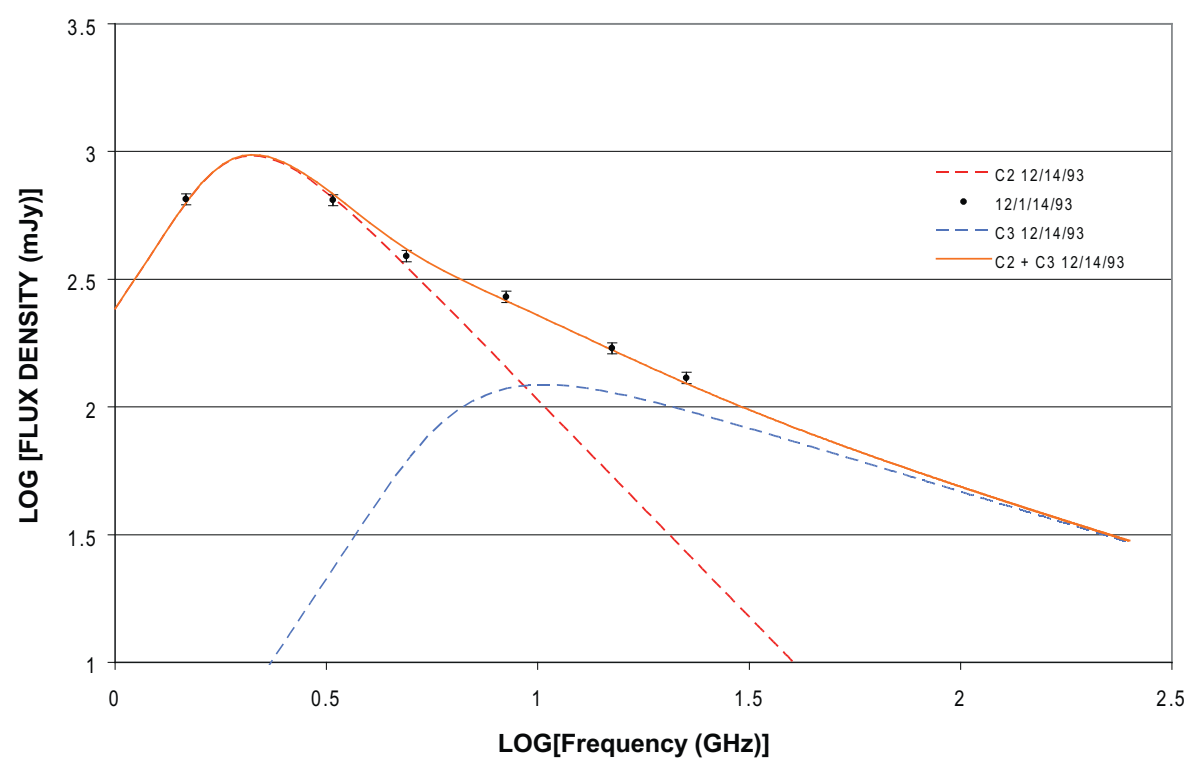

Fig. 4.- The spectrum of GRS 1915+105 on December 14, 1993. Component C1 has evolved to lower frequency and does not appear to contribute noticeably near the local maximum near 2.5 GHz. The 6 data points fix the 6 free parameters in the two synchrotron-self absorbed models of $\mathrm{C} 2$ and $\mathrm{C} 3$. The derived value of $\alpha$ is the same in all three epochs for $\mathrm{C} 2$. 
components. Figures 1 and 2 show the flux density data and some spectral fits on December 6, 1993. Inverted spectra in self-absorbed radio sources are common. However, the inverted spectrum from $1.465 \mathrm{GHz}$ to $3.277 \mathrm{GHz}$ juxtaposed to the steep spectrum, $\alpha=1.9$ (where the convention $L_{\nu}=L_{0} \nu^{-\alpha}$ is assumed), from $3.277 \mathrm{GHz}$ to $4.885 \mathrm{GHz}$ represents very large gradients in $\alpha$ with frequency. Such a large gradient (equivalently, such a narrow local maximum in the spectrum) is extremely unusual for a radio source and provides a strong constraint on the nature of the flare that will be exploited in this section.

\subsection{Synchrotron Self Absorbed Component Models}

The peaked spectral luminosity of ejected plasmoids is generally considered to be a consequence of radiative transfer through a synchrotron self absorbed (SSA) plasma. It is assumed that the emission region is a homogeneous sphere ejected from the central engine. This is certainly more accurate than a continuous jet for emission that shows no evidence in Rodriguez et al (1995); Rodriguez and Mirabel (1999); Rushton et al (2010); Foster et al (1996); Fender et al (1999); Rushton et al (2011) of being quasi-steady. The lack of protracted periods of powerful quasi-steady flux is in contradiction to what would be expected from a large smoothly varying structure, like the jet models in Konigl (1981). Every indication is that the preponderance of the emission on December 6, 1993 is in the form of a large impulsive burst possibly on the background of some low luminosity feature (perhaps the continuous jet). The episodic flaring nature of the source indicated in Rodriguez et al (1995); Pooley and Fender (1997); Rushton et al (2010, 2011) is consistent with large ejections of highly energized plasma, with the epoch December 6, 1993 being one of the most energetic. The epoch December 6, 1993 plasmoid might have an irregular shape with an inhomogeneous density, but without any prior knowledge of these geometric properties, a spherical solid is implemented as a zeroth order approximation. The strategy is to perform the final calculation in the plasma rest frame using known variables from observation. Observed quantities will be designated with a subscript, "o", in the following expressions. Taking the standard result for the SSA attenuation coefficient in the plasma rest frame and noting that $\nu=\nu_{o} / \delta$, we find from Reynolds et al (1996); Ginzburg and Syrovatskii (1969),

$$
\begin{aligned}
& \mu(\nu)=\frac{3^{\alpha+1} \pi^{0.5} g(p) e^{2} N_{\Gamma}}{8 m_{e} c}\left(\frac{e B}{m_{e} c}\right)^{(1.5+\alpha)} \nu_{o}^{(-2.5+\alpha)} \delta^{(2.5+\alpha)}, \\
& g(n)=\frac{\Gamma[(3 n+22) / 12] \Gamma[(3 n+2) / 12] \Gamma[(n+6) / 4]}{\Gamma[(n+8) / 4]}
\end{aligned}
$$

The Doppler factor, $\delta$, is given in terms of $\Gamma$, the Lorentz factor of the outflow; $\beta$, the three velocity of the outflow and the angle of propagation to the line of sight, $\theta ; \delta=1 /[\Gamma(1-$ 
$\beta \cos \theta)$ ] (Lind and Blandford 1985). This equation derives from an assumed powerlaw energy distribution for the relativistic electrons, $N(E)=N_{\Gamma} E^{-n}$, where the radio spectral index $\alpha=(n-1) / 2$ and $E$ is the energy of the electrons in units of $m_{e} c^{2}$. The radiative transfer equation was solved in Ginzburg and Syrovatskii (1969) to yield the following parametric form for the observed flux density, $S_{\nu}$, from the SSA source,

$$
S_{\nu}=\frac{S_{o} \nu^{-\alpha}}{R \mu(\nu)} \times\left(1-e^{-\mu(\nu) R}\right),
$$

where $\mathrm{R}$ is the radius of the spherical region in the rest frame of the plasma and $S_{o}$ is a normalization factor. In the spherical, homogeneous approximation, one can make a simple parameterization of the SSA attenuation coefficient, $\mu(S S A)=\mu_{o} \nu_{o}^{(-2.5+\alpha)}$. If one assumes that the source is spherical and homogeneous then there are three unknowns in equation (3), $R \mu_{o}, \alpha$ and $S_{o}$, and $S_{\nu}$ is determined by observation.

Figure 1 shows the simultaneous VLA data from December 6, 1993. The data seems to indicate three local maxima in the spectrum (one between $1.4 \mathrm{GHz}$ and $5 \mathrm{GHz}$, one near $8.3 \mathrm{GHz}$ and one near $22 \mathrm{GHz}$ ). It should be noted that the SSA function is not an arbitrary shape. For example, it has precisely one maximum. Thus, even though each SSA function has three parameters, there is no guarantee, in general, that two SSA components can fit six or less points exactly. Two SSA components can create at most two local maxima in spectrum. Thus a minimum of three components seems indicated by the simultaneous subset of the data. The comparison between a two component fit and a three component fit is given in Figure 1. The fits are made by letting all the SAA component parameters vary, then minimizing the sum of the squares of the residuals between the simultaneous data and the models. The deviation of the two component model and the data is beyond what is expected based on the statistical uncertainty in the data. Each VLA data point can be treated as an independent statistical variable, $X_{i}, i=1,2,3,4,5$ that is normally distributed with a mean and standard deviation given by the flux density value and the flux density error in Table 1, respectively. The two pints that are more than $1 \sigma$ from the mean only have about a $10 \%$ chance of occurring by random chance and indicate the inability to fit the local maximum in that region. Consider the null hypothesis that the five data points are fit by the model. Each data point provides an independent statistical variable and probability. Therefore, comparing the models in Figure 1 to the data implies that the probability of rejecting the null hypothesis is 0.99 for a two component fit and 0.512 for a three component fit. Thus, the three component fit cannot be rejected on a statistical basis, but the two component fit is rejected with a very high statistical significance. Restricting the discussion to the simultaneous VLA data removes two uncertainties for the fits of the December 6 data in Table 1. First of all, the data is simultaneous, so there is no issue of variability within the short observations. Secondly, the VLA data is very high signal to noise so the errors are 
small (see Table 1) which allows us to exclude a two component model.

In Figure 2, the entire data-set from December 6 is fit by using the three parameter SSA spectrum described above in equation (3) to represent each spectral inflection point. There appears to be three compact components corresponding to the three local maxima. The component that is constrained the least is C3, the high frequency component, since it is highly dependent on the $234 \mathrm{GHz}$ flux density that was taken 12 hours later than the VLA data. The radio flux in GRS 1915+105 can vary significantly in 12 hours (Rodriguez et al 1995; Rushton et al 2010). The other less reliable point is the $3.277 \mathrm{GHz}$ datum from Nancay which was also taken 12 hours after the VLA data. Thus, its exact value was not determinant in fitting component C1. It appears that the flux density was likely increasing near the spectral peak during those 12 hours (the flare was still rising). The fit in Figure 2 uses 9 free parameters to fit 7 data points. The fit that was chosen is not unique. For example, one could pick the fit that minimizes the sum of squares of the errors. Instead, based on the above comments on the 12 hour delay in collecting the $234 \mathrm{GHz}$ and $3.277 \mathrm{GHz}$ data, these data were weighted less heavily in selecting the fit.

Before, discussing the other epochs, it is important to digress on the issue of solving for all three epochs simultaneously and its relationship to the number of total unknowns and the total number of free parameters in the models. The fact that all the free parameters are constrained for C2 and C3 in Figure 4 (epoch December 14, 1993), can be used to remove the arbitrariness of the fits for December 6 and 11 in Table 1. In particular, these values of $\alpha$ for C2 and C3 can be used in the December 6 and 11 fits to the data. Similarly, the steep low frequency spectral index seen in the December 11 data in Figure 3 can be used to determine $\alpha$ in $\mathrm{C} 1$ in both that epoch and on December 6. In summary, using the fact that there is a causal connection between the plasma state in the 3 epochs is being utilized to reduce the total number of free parameters in the 3 epochs from 21 to 17 . There are 17 data points in the three epochs and 17 unknowns which is a well defined solution.

The flare is monitored for a couple of weeks in Rodriguez et al (1995) with varying spectral coverage. Table 2 includes the parameters for the fits to the data from two other epochs of observation of the same flare. On December 11, 1993 and December 14, 1993 there was sufficient spectral coverage to resolve component C2. Figures 3 and 4 show the data from those epochs and the best fits. Clearly there is spectral evolution. The data from December 11, 1993 does not have sufficient high frequency coverage to fit the contribution of component C3. Thus, there is no reference to this component on December 11 in the table. The spectral turnover of component C1 has evolved on December, 11, due to component expansion, so as to fall below the observed frequency range at $1.4 \mathrm{GHz}$ as indicated in the table. On December 14, there is insufficient low frequency coverage to put any constraints 
on component $\mathrm{C} 1$ that had expanded so as to be optically thin far below $1.4 \mathrm{GHz}$ and was apparently very weak at the lowest observed frequencies. Figure 3 has four data points and is fit with six parameters arising from the parametric form (defined in equation 3 ) for the SSA spectra of the components $\mathrm{C} 1$ and $\mathrm{C} 2$. This is reduced to four free parameters by assuming that $\alpha$ is unchanged from December 6 (see the discussion above). In Figure 4, there are 6 data points that are fit by the 6 free parameters associated with SSA spectral fits to the components $\mathrm{C} 2$ and C3. The fits have the same values of $\alpha$ as they had on December 6 .

Figures 1 to 4 show the time evolution of the three components based on the fits at the three epochs. Only component, C2, is adequately sampled so as to be resolved in all three epochs. Figures 1 to 4 do not tell us very much about the evolution of component $\mathrm{C} 1$ since the spectral peak has evolved below the data sampling range by the second epoch. It is clear from Figures 1 to 4 that component $\mathrm{C} 2$ is not adiabatically expanding. If that were true the spectral shape would merely migrate toward lower frequency with some decrease in the peak flux (Moffet 1975). To the contrary, the peak flux density increased dramatically from December 6, 1993 to December 11, 1993. This indicates that the plasmoid is evolving to a state of higher radiative efficiency. The evolution between December 11 and December 14 is closer to adiabatic expansion as the peak flux is approximately constant. Thus, the rate at which the radiative efficiency is increasing slowed down dramatically after December 11. The highest radiative efficiency is in the minimum energy configuration. So, the plasmoid was far from the minimum energy configuration on December 6, but appears to be evolving towards minimum energy after December 11. By contrast, Figure 2 and 4 indicate that component C3 is shifting in frequency and the spectral peak is weakening after December 6. This type of behavior is similar to that of a component evolving very close to adiabatic expansion (Moffet 1975). Note that C3 is a much weaker component than C1 and C2.

\subsection{Spectral Ageing and the Constant $\alpha$ Assumption}

The constant $\alpha$ assumption is required to formally close the set of equations as discussed above. In this subsection, the implications of this assumption in terms of spectral ageing and the generality of the model is discussed.

Note that the mathematical constraint of "constant $\alpha$ " translates physically to the notion that after December 6, the spectral ageing of the components is not dramatic. This does not mean that there was not pronounced spectral ageing before December 6 and modest spectral ageing after December 6. There really is no direct information on the time history of the ejecta before December 6. For example, the different spectral indices of the three components on December 6 is consistent with the components being ejected staggered in 
time: C1 first and C3 last with the spectral steeping occurring from radiation losses. If the spectral ageing mechanism is similar for the three components then in any time snapshot C1 will be steeper than $\mathrm{C} 2$ and $\mathrm{C} 2$ steeper than C3. A simple reverse time evolution of the models found in section 5, indicate substantial spectral ageing must have occurred due to synchrotron losses in a strong internal magnetic field and inverse Compton losses in a strong ambient X-ray field. This is a very plausible explanation of the unusually steep spectral indices of the components $\mathrm{C} 1$ and $\mathrm{C} 2$ from December 6 to December 14. For example, consider the existence of a strong X-ray flare during the time that component C1 was launched that decayed on a time scale of hours. Inverse Compton losses in the first hour after ejection would make the leptons in $\mathrm{C} 1$ cool more than the leptons in $\mathrm{C} 2$ and those in C3 might not be cooled very much at all (because the X-ray flare was weak when C3 was launched). So in principle, for a time variable source, not only is the elapsed time for spectral ageing important, but also the strength of the ambient X-ray background at the time at which the plasma was ejected. Similarly, one might have a scenario in which a magnetic flare in the inner accretion flow launches the jet. The first ejection removes a large fraction of the magnetic flux. Most of the remainder is removed by the ejection of $\mathrm{C} 2$ and C3 has a relatively weak magnetic field. This scenario would also create different spectral ageing rates in the spectral components. The leptons would cool primarily by synchrotron self-Compton emission in the early stages and synchrotron emission later on. The most rapid rate of ageing would also be in $\mathrm{C} 1$. As the plasmoids expand, the rate of spectral ageing would decrease since the magnetic field is getting weaker.

The constant $\alpha$ assumption noted above is tantamount to the following scenario. The plasmoids are ejected and cooled in different ambient X-ray backgrounds, or magnetic environments. In particular, the X-ray background or magnetic field was strong when C1 was launched and decayed during the time interval that subsequent ejecta were emitted. Thus, one day after being ejected, the leptonic energy spectrum of $\mathrm{C} 1$ is steeper than the leptonic energy spectrum of $\mathrm{C} 2$ would be one day after it was ejected. By December 6, the spectral ageing rate is comparatively very slow (a much weaker source of cooling and most of the high energy particles have already been cooled). This "conjecture" is consistent with the models found in section 5. After December 6, the models are shown in section 5 to have higher synchrotron cooling rates than inverse Compton cooling rates. Thus, after December 6 , one would expect that synchrotron cooling is the dominant driver of spectral ageing. The total energy emitted in the synchrotron spectrum between December 6 and December 14 is $\approx 4 \times 10^{39}$ ergs and the total energy stored in the leptons comprising the ejected plasmoids in the models of section 5 is about $\approx 6 \times 10^{42}$ ergs. This indicates that the radiation losses are a negligible contributor to the energy budget of the plasmoids after December 6 . Thus, spectral ageing after December 6 is not be expected to be large, self-consistently with the 
models.

The data on December 11 and 14 is not sufficiently sampled to show that further spectral ageing has or has not occurred after December 6. If indeed there is some spectral ageing, this should not drastically affect the results of the models. The empirical reason is that the basic extreme inflections in the spectral shape drive the model. Namely, the approximate values of $\alpha$ are still determined directly by the data. The data clearly indicates a steep energy index for $\mathrm{C} 1$ and $\mathrm{C} 2$ per the second paragraph of this section. Also, the energy spectrum of C3 cannot be steep due to the $234 \mathrm{GHz}$ point on December 6 and the 22.5 $\mathrm{GHz}$ point on December 14. It must be remembered that because of the constant spectral index expedience that the solutions presented here are not unique. It is however argued that significant departures from the spectral indices used in the analysis are not supported by the data as discussed above. The arguments presented are not a rigorous proof that there is no significant spectral ageing after December 6, but there is no direct evidence to say otherwise.

\section{Kinematic Models of the Plasmoids}

In this section, the fit parameters in Table 2 are related to actual physical parameters. There is a finite range of physical parameters that are consistent with these spectral fits. To make the connection, one needs to relate the observed flux density in equation (3) to the local synchrotron emissivity within the plasma. The synchrotron emissivity is given in Tucker (1975) as

$$
\begin{aligned}
& j_{\nu}=1.7 \times 10^{-21}\left(4 \pi N_{\Gamma}\right) a(n) B^{(1+\alpha)}\left(4 \times 10^{6} / \nu\right)^{\alpha} \\
& a(n)=\frac{\left(2^{\frac{n-1}{2}} \sqrt{3}\right) \Gamma\left(\frac{3 n-1}{12}\right) \Gamma\left(\frac{3 n+19}{12}\right) \Gamma\left(\frac{n+5}{4}\right)}{8 \sqrt{\pi}(n+1) \Gamma\left(\frac{n+7}{4}\right)} .
\end{aligned}
$$

One can transform this to the observed flux density, $S\left(\nu_{o}\right)$, in the optically thin region of the spectrum using the relativistic transformation relations from Lind and Blandford (1985),

$$
S\left(\nu_{o}\right)=\frac{\delta^{(3+\alpha)}}{4 \pi D_{L}^{2}} \int j_{\nu}^{\prime} d V^{\prime},
$$

where $D_{L}$ is the luminosity distance and $j_{\nu}^{\prime}$ is evaluated in the plasma rest frame at the observed frequency. The distance to GRS 1915+105 is debatable with estimates in the range of $6 \mathrm{kpc}$ to $12.5 \mathrm{kpc}$ (Miller-Jones et al 2005). This estimate directly affects the inferred rates that kinematic components are expanding and therefore the determination of $\delta$. We adopt the popular values that Rodriguez et al (1995); Rodriguez and Mirabel (1999) found 
to be consistent with the observations of the flare from March 1994,

$$
D_{L}=12.5 \mathrm{kpc}, \delta=0.57 \text {. }
$$

Combining the results of equations (1) through (7) with the fits in Table 2, we have two equations (constraints) and four unknowns ( $\alpha$ is known through Table 2 ). The constraints are given by frequency of the spectral peak and peak flux density (equivalently, the normalization of the background synchrotron power law and the frequency of unit optical depth defined by $\mu(\nu) R=1)$. The unknowns can be described by $B$, the magnetic field, $N_{\Gamma}$, the normalization of the energy distribution of leptons, $E_{\text {min }}$, the minimum energy of the lepton energy power law and $R$, the radius of the spherical plasmoid. The solution space is illustrated graphically. Figures 5 and 6 are plots of $B$ and $N$, the total number of particles in the plasmoid, as a function of $\mathrm{R}$ for each value of $E_{\min }$ for the three components on December 6, 1993, where

$$
N=\int_{E_{\min }}^{E_{\max }} N_{\Gamma} E^{-n} d E
$$

Five representative values of $E_{\min }$ are considered in the family of models presented in the following that are equal to 1, 5, 10, 20 and 30. The integral in equation (8) is insensitive to the value of $E_{\max }$ for large $n$ as long as $E_{\max } \gg E_{\min }$. A liberal value of $E_{\max }=10^{6}$ was chosen. Reasonable variations in this choice will make modest changes to the energy in C3, since $n=2$ is not large. However, the energy in this component appears to be very reduced compared to the other components, regardless of this choice. The models of C3 are the most poorly constrained because of the sparse high frequency coverage. The trends to note are that $B$ always increases dramatically as the radius of the model of the plasmoid increases for all three components. Furthermore, $B$, is not a function of $E_{\min }$. By contrast, $N$ decreases dramatically as the radius of the plasmoid increases. The total number of particles is very dependent on $E_{\text {min }}$ especially for $\mathrm{C} 1$ and $\mathrm{C} 2$ that have the steep high frequency power laws (equivalently, a steep lepton energy distribution). It is interesting to note the size of the largest component, $\mathrm{C} 1$. The highest resolution ever used to view an ejected plasmoid in a major flare are the $2.0 \mathrm{~cm}$ VLBA observation of Dhawan et al (2000). It is not clear from Dhawan et al (2000) if the $2 \mathrm{~cm}$ observations reveal the emitted component if the data is not tapered. The VLBA observations at $2.0 \mathrm{~cm}$ are capable of resolving components $R>2 \times 10^{14}$ $\mathrm{cm}$. Thus, only the largest models of $\mathrm{C} 1$ could be marginally resolved by the VLBA and C2 and C3 could not be resolved on December 6. The Dhawan et al (2000) observations of two different flares were unable to resolve the ejected region of high emissivity in the image plane either with full resolution at $3.6 \mathrm{~cm}$ or with tapering at $2 \mathrm{~cm}$, consistent with the predications 
of the models presented here. Thus, the plasmoids that are considered here are too small to have their sizes accurately fit with state of the art interferometry, except possibly if they are observed at very high frequency with VLBA.

One can also look at the time evolution of $B$ and $N$ for component C2. Figures 7 and 8 plot $B$ and $N$, respectively, for the allowed plasmoids models of $\mathrm{C} 2$ at all three epochs. Figures 7 and 8 show that the plasmoid is expanding for any reasonable value of $B$ and $N$. Furthermore, Figure 7 indicates that $B$ is decreasing as the plasmoid propagates away from the central engine. Again, on December 14, C2 is still too small to be resolved except marginally with high frequency with VLBA.

\section{Ramifications of a Protonic Model}

In order to discuss, the possibility of protonic plasmoids versus positronic plasmoids, one needs to separate the energy content into two pieces. The first is the kinetic energy of the protons, $E$ (proton). The other piece is named the lepto-magnetic energy, $E(\mathrm{~lm})$, and is composed of the volume integral of the leptonic thermal energy density, $U_{e}$, and the magnetic field energy density, $U_{B}$. It is straightforward to compute the lepto-magnetic energy in a spherical volume from the solutions in Figures 5 to 8 for $B$ and $N$,

$$
E(\operatorname{lm})=\int\left(U_{B}+U_{e}\right) d V=\frac{4}{3} \pi R^{3}\left[\frac{B^{2}}{8 \pi}+\int_{1}\left(m_{e} c^{2}\right)\left(N_{\Gamma} E^{-n+1}\right) d E\right]
$$

The kinetic energy of the protonic component is

$$
E(\text { protonic })=(\Gamma-1) M c^{2},
$$

where $\Gamma$ is the bulk Lorentz factor and $M$ is the mass of the plasmoid. There is no high resolution multi-epoch radio imaging of the ejected plasmoids for the December 1993 flare, so one cannot estimate these kinematic values directly from observation. However, GRS 1915+105 has shown fairly similar kinematics of the ejected plamsoids for the various flares that have been monitored since 1994 (Mirabel and Rodriguez 1994; Rodriguez et al 1995; Fender et al 1999; Dhawan et al 2000; Miller-Jones et al 2005). The kinematic fits of the plasmoid motion for the March 1994 major flare indicate that the motion is consistent with a bulk velocity is $v=0.92 c$ and the Lorentz factor associated with this motion is $\Gamma=2.55$ (Mirabel and Rodriguez 1994; Rodriguez and Mirabel 1999). The values are not that much different than those inferred for other major flares (Fender et al 1999; Rodriguez and Mirabel 1999). In Fender (2003), it was noted that these kinematic estimates can only be considered lower limits to $\Gamma$. However, these lower limits are not necessarily 
Table 1: Flux Densities of GRS 1915+105

\begin{tabular}{cccccc}
\hline Date & $\begin{array}{c}\text { UT Time } \\
\text { (days) }\end{array}$ & $\begin{array}{c}\text { Frequency } \\
(\mathrm{GHz})\end{array}$ & $\begin{array}{c}\text { Flux Density } \\
\text { mJy }\end{array}$ & $\begin{array}{c}\text { Radio } \\
\text { Telescope }\end{array}$ & $\begin{array}{c}\text { Error } \\
\%\end{array}$ \\
\hline $12 / 6 / 93$ & 0.02 & 1.465 & 209 & VLA-D & 5 \\
$12 / 6 / 93$ & 0.59 & 3.277 & 1083 & Nancay & 5 \\
$12 / 6 / 93$ & 0.02 & 4.885 & 503 & VLA-D & 5 \\
$12 / 6 / 93$ & 0.03 & 8.415 & 398 & VLA-D & 5 \\
$12 / 6 / 93$ & 0.03 & 14.965 & 241 & VLA-D & 5 \\
$12 / 6 / 93$ & 0.03 & 22.485 & 239 & VLA-D & 5 \\
$12 / 6 / 93$ & 0.60 & 234.000 & 123 & IRAM & 10 \\
\hline $12 / 11 / 93$ & 0.57 & 1.407 & 1319 & Nancay & 5 \\
$12 / 11 / 93$ & 0.38 & 2.695 & 860 & Bonn & 10 \\
$12 / 11 / 93$ & 0.57 & 3.277 & 901 & Nancay & 5 \\
$12 / 11 / 93$ & 0.50 & 10.550 & 403 & Bonn & 10 \\
\hline $12 / 14 / 93$ & 0.56 & 1.407 & 653 & Nancay & 5 \\
$12 / 14 / 93$ & 0.60 & 1.410 & 615 & Bonn & 10 \\
$12 / 14 / 93$ & 0.85 & 1.465 & 650 & VLA-D & 5 \\
$12 / 14 / 93$ & 0.56 & 3.277 & 646 & Nancay & 5 \\
$12 / 14 / 93$ & 0.85 & 4.885 & 390 & VLA-D & 5 \\
$12 / 14 / 93$ & 0.85 & 8.415 & 270 & VLA-D & 5 \\
$12 / 14 / 93$ & 0.85 & 14.965 & 170 & VLA-D & 5 \\
$12 / 14 / 93$ & 0.85 & 22.480 & 130 & VLA-D & 5
\end{tabular}

Table 2: Description of Synchrotron-Self Absorbed Components

\begin{tabular}{ccccccc}
\hline Component & Date & $\alpha$ & $S_{o}\left(\mathrm{ergs} / \mathrm{cm}^{2}-\mathrm{sec}\right)$ & $R \mu_{o}$ & Peak $(\mathrm{GHz})$ & Peak $(\mathrm{mJy})$ \\
\hline $\mathrm{C} 1$ & $12 / 6 / 93$ & 2.2 & $9.65 \times 10^{-3}$ & $5.30 \times 10^{-4}$ & 2.9 & 857 \\
$\mathrm{C} 1$ & $12 / 11 / 93$ & 2.2 & $1.82 \times 10^{-3}$ & $\ldots$ & $<1.4$ & $\ldots$ \\
$\mathrm{C} 2$ & $12 / 6 / 93$ & 1.7 & $2.60 \times 10^{-7}$ & $1.50 \times 10^{-1}$ & 9.5 & 245 \\
$\mathrm{C} 2$ & $12 / 11 / 93$ & 1.7 & $3.12 \times 10^{-7}$ & $5.43 \times 10^{-3}$ & 5.5 & 826 \\
$\mathrm{C} 2$ & $12 / 14 / 93$ & 1.7 & $7.70 \times 10^{-8}$ & $2.54 \times 10^{-4}$ & 2.6 & 705 \\
$\mathrm{C} 3$ & $12 / 6 / 93$ & 0.5 & $5.70 \times 10^{-19}$ & $1.00 \times 10^{1}$ & 49.0 & 224 \\
$\mathrm{C} 3$ & $12 / 14 / 93$ & 0.5 & $1.47 \times 10^{-19}$ & $1.16 \times 10^{-1}$ & 13.5 & 108
\end{tabular}


inaccurate in every instance. With this important disclaimer in mind, the popular March 1994 values from Mirabel and Rodriguez (1994) for the December 1993 flare are adopted in the following.

Figure 9 plots the energy content of component C1 on December 6, 1993 for each member of the family of models. The lepto-magnetic energy, $E(\mathrm{~lm})$, is plotted as a dashed curve for each value of minimum energy in the adopted models $1<E_{\text {min }}<30$ as function of the modeled plasmoid radius, $R$. The total energy, $E$ (Total) $=E(\operatorname{lm})+E$ (protonic), is plotted as a solid line. The curves are color coded, so $E(\mathrm{~lm})$ and $E$ (Total) are the same color for each value of $E_{\text {min }}$. For example, the $E_{\text {min }}=1$ curves are green. First note that the minimum of $E(\mathrm{~lm})$ and $E$ (Total) are at very different modeled plasmoid radii. The minimum of $E(\mathrm{~lm})$ occurs near the equipartition point, $U_{B} \gtrsim U_{e}$. This is indicated by the vertical dashed green line for $E_{\min }=1$. To the left of this line, $U_{B}<U_{e}$, and to the right of this line $U_{B}>U_{e}$. Note that the vertical continuation of the dashed line to its intersection with the $E$ (Total) curve is far to the left of the minimum of the $E$ (Total) curve. Conversely, the minimum of the $E$ (Total) curve occurs for models in which $U_{B} \gg U_{e}$. One can infer that $E$ (Total) is dominated by the protonic kinetic energy except for large radii where the number density is small and the magnetic energy density is large (i.e., the lepto-magnetic energy is much smaller than the protonic kinetic energy unless the plasmoid is extremely magnetically dominated). For extreme magnetic dominance, $E($ Total $) \approx E(\operatorname{lm})$, at large $\mathrm{R}$.

For a protonic plasma, one can compute the spatially averaged energy flux in the plasmoid, $<Q>$, as

$$
<Q(\text { protonic })>=(1 / 2 R) \int_{-R}^{R} \pi\left(R^{2}-r^{2}\right) \Gamma(\Gamma-1) n v m_{p} c^{2} d r .
$$

A spatially averaged energy flux is calculated because the geometry is spherical, not conical, by assumption. The geometrical cross-section of the sphere that is orthogonal to the plasmoid bulk velocity vector is zero at the poles and maximal at the equator. The energy flux scales with this cross-sectional surface area, thus there is major variation of the energy flux along the polar diameter. Thus, an average over the polar diameter (projected along the direction of the bulk velocity vector) gives a more appropriate quantity, the spatially averaged energy flux. The $\pi\left(R^{2}-r^{2}\right)$ term in equation (11) is the cross sectional area of the spheroid that is orthogonal to the bulk velocity vector, as $r$ varies from the north pole at $r=-R$ to the south pole at $r=R$. Similarly, for the lepto-magnetic component of the plasma, one can compute the spatially averaged energy flux in the plasmoid, $<Q(\operatorname{lm})>$, as

$$
<Q(\operatorname{lm})>=(1 / 2 R) \int_{-R}^{R} \pi\left(R^{2}-r^{2}\right)\left(\Gamma^{2}\right) v\left(U_{B}+U_{e}\right) d r .
$$


The total energy flux, $Q$ (Total) $=<Q(\operatorname{lm})>+<Q$ (protonic) $>$. This measure of energy flux can be distinguished from the energy flux injected into the plasmoid from the central engine. In order to assess the origin of the plasmoids, it is much more revealing to look at the power required to initiate the plasmoid, $Q_{\text {protonic }}($ injected), rather than the redistribution of energy flux within the plasmoid, far from the central engine. The power injected by the central engine is defined by

$$
Q_{\text {protonic }}(\text { injected })=E(\text { protonic }) / t_{\text {rise }}=(\Gamma-1) M c^{2} / t_{\text {rise }},
$$

where $t_{\text {rise }}$ is the amount of time for the energy to be injected into the plasmoid, the flare rise time. Similarly, for the lepto-magnetic energy flux that is injected

$$
Q_{\operatorname{lm}}(\text { injected })=E(\operatorname{lm}) / t_{\text {rise }} \text {. }
$$

The radio flux density during the rise of the flare was not sampled during the December 1993 flare. There is high time resolution radio sampling of four major flares that was found in the archival literature that is plotted in Figure 10. The four flares with rise time coverage were all monitored at $15 \mathrm{GHz}$ with the Ryle telescope in Rushton et al (2010). Two of the flares were actually well sampled at $15 \mathrm{GHz}$ in Rushton et al (2010), the 1997 flare on MJD 50752 and the 1999 flare on MJD 51337. The two 1998 flares in Figure 10 were not well sampled on their rise at $15 \mathrm{GHz}$ in Rushton et al (2010). Fortunately, there is excellent data sampling on the rise time at $2.3 \mathrm{GHz}$ and $8.4 \mathrm{GHz}$ for the 1998 flares on MJD 50917 and MJD 50934 in Dhawan et al (2000). The $8.4 \mathrm{GHz}$ data is used to estimate $t_{\text {rise }}$ for these flares. There is also a crude estimate on $t_{\text {rise }}$ for the $1.4 \mathrm{GHz}$ flux from the March 1994 flare of $12 \pm 5$ hours that was derived in Rodriguez et al (1995) by extrapolating the motion of VLA components backwards in time combined with sparsely sampled radio monitoring. This point with its large error bars is also added to the plot. The figure indicates that stronger flares tend to have larger $t_{\text {rise }}$. The strongest flare from March 1994 may not follow the linear trend that fits the other 4 flares very well - the error bars are too large to make this determination. The linear fit for the other four data points is

$$
t(\text { rise }(\text { hours }))=2.981+0.0191 S_{\nu}(15 \mathrm{GHz})
$$

with a coefficient of determination of $R^{2}=0.9819$. Thus, it seems reasonable to use archival data to estimate the rise time of the December 1993 flare. The December 1993 flares C1 and $\mathrm{C} 2$ have $15 \mathrm{GHz}$ flux densities similar to the MJD 50752 and MJD 50934 flares, so $t_{\text {rise }} \approx 7.5 \mathrm{hrs} \approx 2 \times 10^{4} \mathrm{sec}$ is chosen as a fiducial $t_{\text {rise }}$. The resultant $Q$ values from equations (15) and (16) can be simply scaled with different choices of $t_{\text {rise }}$. The uncertainty here, based on monitored flares in the rising phase seems to be a factor of 2 at most. 
Figure 9 contains a plot of $Q$ (Total) and $Q(\mathrm{~lm})$ based on the values of $E$ in Figure 9 divided by $t_{\text {rise }}=2 \times 10^{4} \mathrm{sec}$. First note that the $Q$ (Total) that is needed to initiate the flare in Figure 9 is larger than the spatially averaged value of $Q$ (Total) in the plasmoid when it was observed on December 6. This is expected because the plasmoid will expand spreading the energetic particles over a larger volume, thereby lowering the average energy flux, $<$ $Q($ Total $)>$. Notice the color coded vertical dashed lines in Figure 9. These lines connect the minimum of the lepto-magnetic energy (commonly referred to as the "minimum energy configuration" in the literature) to the corresponding $Q$ (Total) for the same numerical model (determined by $R$ and $E_{\min }$ ). For $E_{\min }=1$, the total injection energy of the "minimum energy solution" is $Q$ (Total) $>10^{43} \mathrm{ergs} / \mathrm{sec}$ which is comparable to the strongest radio flares in the powerful nearby quasar Mrk 231 Reynolds et al (2009). This seems very unlikely. Even the "minimum energy solution" for $E_{\min }=5$ has $Q$ (Total) $>10^{41} \mathrm{ergs} / \mathrm{sec}$, comparable to many radio quiet quasars and their galactic hosts. Thus, Figure 9 shows us that the combination of the minimum energy assumption and $E_{\min }<5$ is not plausible for the $\mathrm{C} 1$ plasmoid, if the plasma is protonic.

One can use Figure 11 to restrict the solution space further. The idea of a protonic plasma seems natural if the constituent matter comes from the accretion flow. The large energy fluxes of the minimum energy solutions that were noted in regards to Figure 9 are problematic for these models. a possible resolution are solutions that are far from minimum energy. A large magnetic field (very non-equipartition) ameliorates the problem associated with an enormous protonic component. However, there is a connection between the observed emission at low frequency $(1.4 \mathrm{GHz})$, the energy of the synchrotron emitting leptons, $E(1.4 \mathrm{GHz}), B$ and the constraint $E>E_{\min }$. Our fits to the $\mathrm{C} 1$ spectra indicate that the $1.4 \mathrm{GHz}$ emission is inherently strong, but it is self-absorbed in the magneto-plasma. Using the formula from Tucker (1975), one can compute the electron energy required for a peak synchrotron flux at an observed frequency, $\nu_{o}$, for a given magnetic field $B$,

$$
E_{\mathrm{peak}} \approx\left[\frac{\nu_{o}(\mathrm{peak}) / \delta}{3 \times 10^{6} B}\right]^{0.5}>E_{\text {min }}, \quad E(1.4 \mathrm{GHz}) \equiv\left[\frac{1.4 \mathrm{GHz} / \delta}{3 \times 10^{6}}\right]^{0.5} .
$$

It is instructive to look at the restriction $E(1.4 \mathrm{GHz})>E_{\min }$ applied to $Q$ (Total) in Figure 11. There is no restriction within the range plotted for $E_{\text {min }}=1$. However, there is one for the other values of $E_{\min }$ that is indicated by the color coded vertical divider and the arrow pointing towards the allowable region of solution space. Solutions that are allowed by equation (16) are to the left of the divider (the direction that the arrow points), solutions that are excluded by this constraint are to the right of the divider. The minimum allowed values of $Q$ (Total) exceed the maximum sustained energy flux in the radiation field from the accretion flow of $\sim 3 \times 10^{38} \mathrm{ergs} / \mathrm{sec}$ from GRS $1915+105$, both contemporaneous with the flare and historically (Harmon et al 1997). Larger soft X-ray (possibly thermal) luminosity has been 
observed, but only very briefly, not long enough to launch the plasmoid (Mirabel et al 1998). There is a small region of solution space with energy fluxes comparable with the luminosity of these high states of sustained thermal X-ray emission, $E_{\text {min }}=20$ restricted to a very small range of $R$ where $U_{B} \gg U_{e}$. It seems very unlikely that disk radiation pressure can provide the force that is necessary to initiate the ejection of the protonic plasmoids. The implication is that magnetic forces would likely be required to energize the protonic plasmoid and eject it at relativistic speeds.

There is more information that can be gleaned from the protonic models of component $\mathrm{C} 2$ as they evolve in time. This is captured in Figure 12. This figure compares the solution space of the $E_{\text {min }}=1$ and $E_{\text {min }}=5$ solutions at two epochs, December 6 and December 11. The solutions for other values of $E_{\min }$ were left off the chart, so as not to clutter it, but the trends that will be described apply to all solutions. These plots are formatted differently than what has been shown previously. This is a plot of $E(\operatorname{lm})$ versus $N$, the total particle number in the plasmoid (instead of $R$ ). As the plasmoid evolves, one expects the baryon number to be conserved. There is no reason to presume significant entrainment both on dynamical grounds since Kelvin-Helmholtz instabilities are inefficient means of mixing media for relativistic flows, Bicknell (1994), and empirically the plasmoids in major flares do not seem to decelerate noticeably from scales of 1 mas to 100 mas (Dhawan et al 2000). Applying baryon conservation to $\mathrm{C} 2$ in Figure 12, shows that as time evolves, the magnetic energy in the system must increase. The vertical dashed lines are drawn to help visualize this. The data is color coded. The $E_{\text {min }}=1$ data is orange both on the December 6 curve (dashed) and the December 11 curve (solid). Similarly, the $E_{\min }=5$ data is purple both on the December 6 curve (dashed) and the December 11 curve (solid). The vertical dashed lines connect solutions with conserved baryon number in time. The dashed lines are chosen to intersect the four minimum energy solutions: $E_{\text {min }}=1$, December $6 ; E_{\min }=1$, December $11 ; E_{\text {min }}=5$, December 6 ; and $E_{\min }=5$, December 11 . Consider the vertical line farthest to the right that intersects the minimum energy configuration at $E_{\min }=1$, December 11 . Baryon conservation, implies that the solution is far to the right of the minimum energy point on the plot at for $E_{\text {min }}=1$ on December 6 , where $U_{e} \gg U_{B}$. Thus, the plasmoid is evolving from a state of $U_{e} \gg U_{B}$ to one of $U_{e} \approx U_{B}$ in 5 days. Since radiation losses are negligible in 5 days $\left(\sim 10^{39}\right.$ ergs) compared to the mechanical energy in the plasmoid, energy conservation implies that mechanical energy is being converted to magnetic energy. In particular, the magnetic energy increases from $2.69 \times 10^{40}$ ergs to $5.08 \times 10^{42}$ ergs in 5 days in these protonic solutions.

One can synthesize the deductions from Figures 9, 11 and 12 as follows:

1. Figure 9 shows that the kinetic luminosity of the protonic solutions can rival quasars 
unless the system is far from equipartition (specifically, it is magnetically dominated, $\left.U_{B} \gg U_{e}\right)$ and $E_{\min } \gg 1$.

2. Figure 11 shows that the protonic solutions must be initiated by extremely large injection energy fluxes, so they cannot be driven by radiation pressure. Furthermore, as noted above, the plasmoid must be magnetically dominated in order to avoid unrealistic energy fluxes. Thus, magnetic forces are required to initiate the protonic plasmoids.

3. Figure 12 shows that mechanical energy is converted to magnetic energy in the protonic plasmoids as they evolve in time

Points 2 and 3 are inconsistent in a straightforward interpretation. Point 2 is that the source of energy for an ejection is magnetic energy that is converted into mechanical form (kinetic energy and heat) when the plasmoid is launched. Point 3 says that the opposite must be true as the plasmoid propagates, magnetic energy is being created from mechanical energy. Thus, there is no causal mechanism to initiate and maintain the protonic plasmoid ejections with a monotonic transfer of energy. One could imagine more elaborate scenarios that are not monotonic. Ejecta begin magnetically dominated. The energy is converted to mechanical form close to the source then begins to convert back to magnetic form farther out. This strong energy transfer at small distance must occur on the orders of hours or less after the ejecta form. Such an abrupt change in the physical state seems to be representative of a shock. Observationally, there is presently no evidence of the entropy generated by such shocks (radiation). Theoretically, this is not favored either. There are two possibilities in MHD, a fast shock and a slow shock depending on whether the flow upstream of the shock front is above or below the Alfven speed, which is near the speed of light in the magnetically dominated limit (Kantrowitz and Petschek 1966). In the magnetically dominated limit, strong fast shocks are inefficient at converting magnetic energy to mechanical energy (Kennel and Coroniti 1984). Conversely, slow shocks are very efficient at converting magnetic energy to mechanical energy. However, downstream of the shock front the flow is necessarily slower than the sound speed (Kantrowitz and Petschek 1966; Punsly 2008). This conflicts with all observations of major flares. The VLBA observations of superluminal velocity for different major flares indicate that the ejecta are relativistic even on small scales (within hours of ejection) Dhawan et al (2000). Thus, a non-monotonic scenario to make points 2 and 3 compatible is difficult to reconcile with observation and MHD.

The nonexistence of protonic plasmoids in GRS 1915+105 that follows from a synthesis of points 2 and 3 also resolves the paradox of the huge implied energy flux described in point 1. It is interesting to note that a study of large IR flares in Fender and Pooley (2000) determined that a protonic jet would require similarly large energy fluxes, $Q \approx 8 \times 10^{42}$ ergs/sec. They also considered this very unlikely. 


\section{Leptonic Plasmoids}

The results of the last section indicate that physical models of the December 1993 flare should be based on electron-positron plasmas, not electron-proton plasmas. This section is a formal investigation of these models. Figure 13 shows the lepto-magnetic energy stored in each component in the models as a function of the plasmoid radius, $R$, for the December 6 data. If one concentrates on the minimum energy solutions then there is a curious trend that the more compact components (younger components) have less energy. To investigate whether this is plausible, one can also plot the lepto-magnetic energy of the component C2 as a function of $R$ at the three different epochs in Figure 14. Note that by December 14, the plots of the energy stored in $\mathrm{C} 2$ are very similar to those of $\mathrm{C} 1$ on December 6 . This suggests that the energy of the flares does not depend on the size on December 6 and this apparent trending with size is only an artifact of the minimum energy assumption.

Figure 14 can also be used to explore the deviations from the minimum energy assumption that were noted above. Horizontal lines connect solutions of the same stored energy at different times. The assumption of conserved energy seems reasonable since the radiative losses during the 8 days of component evolution are negligible $\left(\sim 10^{39} \mathrm{ergs}\right)$. 1 The plots of the energy stored in the models of the plasmoid change mildly from December 11 to December 14, unlike the drastic changes seen between December 6 and December 11. This indicates that the evolutionary rate of change of the constituent physical parameters has slowed considerably by December 14. Furthermore, the radiative efficiency is approaching a maximum on December 14 as noted in regards to Figures 1 to 4 . The slowing rate of change of the physical parameters indicates that the system is close to an equilibrium, near maximum radiative efficiency (the state of the plasmoid is slowly approaching the minimum energy configuration). Choosing the December 14 state as approximately the minimum energy state seems reasonable since this is the only natural equilibrium state of the system (of course, there could be unknown, more complicated, dynamical principles that drive the plasmoid toward a different equilibrium or an approach to some other asymptotic state). This motivates the detailed study of the evolution towards the minimum energy state that follows in this section. The results can be applied to other asymptotic states near equipartition without any loss of generality.

One can see the consequences of approximate energy conservation in the context of the asymptotic minimum energy assumption, by tracing the color coded horizontal dashed lines in Figure 14. These lines connect similarly color coded solution spaces for a particular

\footnotetext{
${ }^{1}$ There also does not seem to be any evidence of significant adiabatic expansion energy losses in Figure 14, so the work done by the plasmoid expanding against an external medium seem to be negligible as well.
} 
value of $E_{\text {min }}$ at the various epochs. For example, the blue dashed line connects the three blue $E_{\text {min }}=1$ solutions at the three different epochs maintaining a constant energy. There are two evolutionary tracks through the solution space. One is inertially dominated on December 6 in which mechanical energy is converted to magnetic energy in time, until the system is near equipartition on December 14. This was the nature of the pathological protonic time dependent solutions that were found in Section 4. The other time dependent solution, is one in which the plasmoid is magnetically dominated on December 6 and magnetic energy is converted into leptonic energy in time so that by December 14, the system is near equipartition. One can distinguish between these two paths through the solution space by considering two items, the possible power sources and the exchange of energy between magnetic and mechanical form during the course of the plasmoid evolution.

There are two plausible mechanisms to power the relativistic plasmoid ejections. First, there is radiation or gas pressure from the disk and the second is magnetic force. Figure 14 indicates that a minimum energy configuration on December 14 evolves from a state that is far from minimum energy on December 6. On December 6, most of the energy is either in pair plasma or magnetic energy. If the plasmoid is powered by radiation or gas pressure from the protonic disk, it does not follow that it would create a highly energetic pair plasmoid. One would expect it to be made of the same matter as its source. However, a model of pair plasma ejected by the disk and accelerated by the radiation field was considered in Liang and Li (1995). The magnetic field was not small initially, but was required by the equations of motion to be significant in order to accelerate the leptons to relativistic energies. However, note that the kinetic luminosity in Table 3 for $E_{\text {min }} \sim 6$ is sufficiently small so as to be consistent with the maximum sustainable radiation pressure from the X-ray luminosity given in Section $4, Q<3 \times 10^{38} \mathrm{ergs} / \mathrm{sec}$. Thus, the solution branch that begins with the plasmoid extremely mechanically dominated on December 6 , does not have enough magnetic field to energize the electrons, but there is enough force from radiation pressure to drive the asymptotic minimum energy solutions, in principle. This solution might be viable if a suitable acceleration mechanism in a pair plasma for the leptons were found. Note that equation (16) implies that a weak magnetic field requires large values of $E$ to produce flux above $10 \mathrm{GHz}$. So, the means of accelerating the leptons is a serious technical difficulty with the model, not just a pedantic one. The new deduction of the models presented here is that the late time solution along the inertially dominated branch of solution space did not evolve from one of significant magnetic field (no lepton acceleration mechanism), so the Liang and Li (1995) solution does not describe the December 1993 flare.

If the relativistic plasmoids are magnetically initiated then it follows that the plasmoids should be magnetically dominated at early times. A scenario in which a plasmoid starts out magnetic then converts to pair plasma then back to equipartition seems very contrived and 
has no known physical basis. The only causal scenario for plasmoid evolution is one in which an energetic plasmoid is created by magnetic forces. The magnetically dominated object dissipates its energy into violent shocks, nonlinear MHD waves and high power reconnection events, thereby converting magnetic energy into a relativistically hot pair plasma.

Thusly motivated, one can explore the magnetically dominated time evolution track through solution space in detail. Figure 15 is a plot of the radius of $\mathrm{C} 2$ versus time for the magnetic solutions that conserve energy and approach the minimum energy solution on December 14 as indicated in Figure 14. The solutions follow a track of uniform expansion very closely. The time offset was adjusted in such a way that all the solutions reach approximately zero radius at time zero. All values of $E_{\min }$ yield virtually the same offset, indicating that the plasmoid was 1.6 days old on December 6 . For $E_{\min }=1,5,10,20$, 30, the least squares fit to the expansion rate, $d R / d t$, is $0.0085 \mathrm{c}, 0.0082 \mathrm{c}, 0.0078 \mathrm{c}, 0.0069 \mathrm{c}$ and $0.0064 \mathrm{c}$, respectively.

One can perform an analysis similar to that which was done for Figure 11 for the protonic plasmoids using equation (16) to restrict the allowable range of $E_{\min }$. Figure 14 is used to illustrate this and also plot the power, $Q(\operatorname{lm}) \equiv Q_{\operatorname{lm}}$ (injected), required by the central engine to energize and eject the lepontic plasmoids at relativistic velocities, where the conversion from $E$ is given by equation (14). The plots of $Q(\mathrm{~lm})$ are color coded so that each model is the same color at all three epochs. Recall that the restriction discussed in Section 4 arises because large values of $B$ require small values of $E(1.4 \mathrm{GHz})$ to create a synchrotron peak near $1.4 \mathrm{GHz}$. The spectral fits indicate that the $1.4 \mathrm{GHz}$ flux is large and only appears suppressed because of synchrotron-self absorption (i,e, the background power law spectrum should extend lower than $1.4 \mathrm{GHz})$. The restriction equates to $E(1.4 \mathrm{GHz})>E_{\text {min }}$. As for Figure 14, the color coded short vertical lines and arrows indicate the radius of the model such that $B$ is sufficiently large that equation (16) implies, $E(1.4 \mathrm{GHz})=E_{\text {min }}$. The arrow points to the left, the region of smaller $B$, where $E(1.4 \mathrm{GHz})>E_{\min }$ and the solutions are allowed. To the right of the vertical partition, $B$ is larger, therefore $E(1.4 \mathrm{GHz})<E_{\min }$ and the solutions are forbidden. These partitions were added to the December 6, 1993 plots. In the the evolutionary track in which magnetic energy is converted to mechanical energy, the magnetic field is largest at this epoch and therefore more likely to provide the tightest constraint on $E_{\min }$. In summary, the solution space is restricted by three constraints,

1. The solution is magnetically dominated on December 6

2. $E(1.4 \mathrm{GHz})>E_{\min }$

3. Energy conservation with the December 14 solution, $E($ December 14$)=E($ December 11$)=$ $E($ December 6) 
Figure 14 shows that the $E_{\min }=20$ and $E_{\text {min }}=30$ allowable solutions on December 6 have lower energy than the minimum energy solution on December 14 unless they are dominated by pair plasma inertia (far to the left of the energy minimum on December 6 ). This violates condition 1 above, so these solutions do not appear to be physical. The $E_{\text {min }}=10$ allowable solutions on December 6 can be magnetically dominated as indicated by the location of the black arrow (to the right of the minimum energy). However, the black arrow is below the black horizontal dashed line indicating that the energy flux of these solutions is below that of the minimum energy solution on December 14, so they would not satisfy energy conservation (violates condition 3 above). Thus, there are no physically allowed solutions with $E_{\text {min }}=10$. The red vertical arrow for the $E_{\text {min }}=5$ allowable solutions lies above the horizontal red dashed line, so there is an allowed solution that is magnetically dominated on December 6 and the energy is conserved until December 14. A careful numerical calculation shows that the largest value of $E_{\min }$ that is allowed by these arguments is $E_{\text {min }}=6$. Note that departures from minimum energy on December 14, will only drop the maximum allowed value of $E_{\text {min }}$ below 6 . So, this argument is more general than one associated with just an asymptotic minimum energy state. The maximum allowed value of $E_{\min }$ is 6 for any of the possible magnetically dominated tracks through the solution space in Figure 14 (i.e., other horizontal tracks that are not indicated by the dashed lines).

Table 3 displays the parameters of solutions for $\mathrm{C} 2$ that approach minimum energy on December 14 and for $\mathrm{C} 1$ on December 6. The table is a useful device for understanding the time evolution of the plasmoid $\mathrm{C} 2$ and its relationship to $\mathrm{C} 1$ at late times. Columns 1 and 2 in Table 3 identify the flare. The next two columns are $E_{\text {min }}$ and the designation of the solution as minimum energy or not, respectively. Columns 5 and 6 are the radius of the plasmoid model and the magnetic field strength, respectively. Column 7 is the ratio of magnetic energy density (trivially computable from column 6) to leptonic energy (i.e., the degree of magnetic dominance). Column 9 is the total number of particles in the plasmoid. The last column is the power required to energize and eject the plasmoid at relativistic speed from the central engine as defined in equation (14).

The component sizes in Table 3 are much smaller than normally assumed in the literature and compactness might be an issue. There might be significant inverse Compton emission that could affect the basic assumptions of the model or further constrain the models. A simple measure of the significance of strong inverse Compton cooling is the brightness temperature, $T_{b}$. These are evaluated for the various models in column (8) of Table 3 by the following method. Physically, it is $T_{b}$ evaluated in the frame of reference (the intrinsic brightness temperature) of the plasmoid, $\left(T_{b}\right)_{p}$, that is relevant for assessing a possible "inverse Compton catastrophe" (Kellermann \& Pauliny-Toth 1969; Marscher et al 1979). We want to express this in terms of observable quantities at earth designated by the subscript "o." First of all, the 


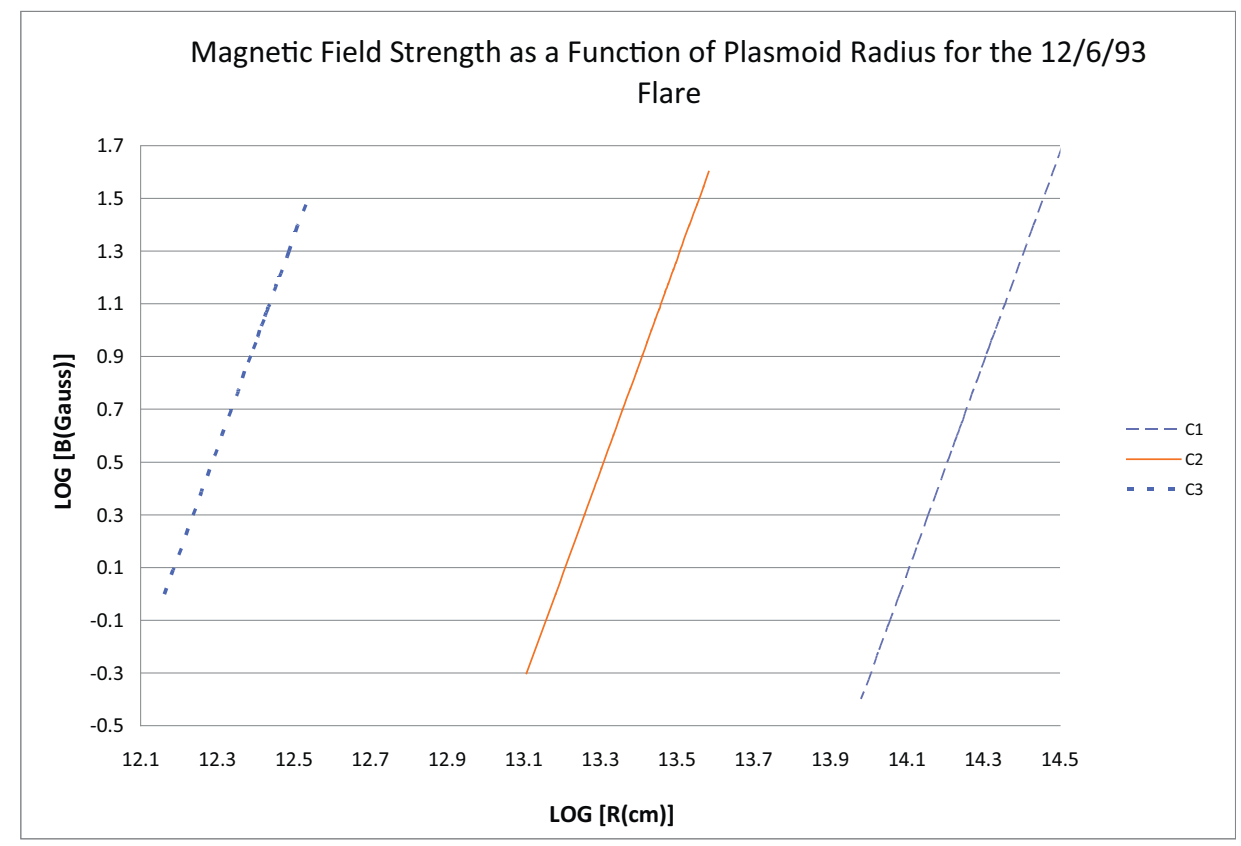

Fig. 5.- The magnetic field strength, $B$, as a function of the radius of the model for each of the three components on December 6 . Notice that $B$ increases with increasing $R$.

Table 3: Parameters of Models that Approach Minimum Energy at Late Time Models

\begin{tabular}{|c|c|c|c|c|c|c|c|c|c|}
\hline Component & Date & $E_{\min }$ & $\begin{array}{l}\text { Minimum } \\
\text { Energy }\end{array}$ & $\begin{array}{c}R \\
\mathrm{~cm}\end{array}$ & $\begin{array}{c}B \\
\text { Gauss }\end{array}$ & $U_{B} / U e$ & $\begin{array}{c}T_{b} \\
10^{11 \circ} \mathrm{K}\end{array}$ & $N$ & $\begin{array}{c}Q \\
\mathrm{ergs} / \mathrm{sec}\end{array}$ \\
\hline C1 & $12 / 6 / 93$ & 1 & Yes & $1.72 \times 10^{14}$ & 4.2 & 1.27 & 1.91 & $4.93 \times 10^{49}$ & $6.14 \times 10^{38}$ \\
\hline $\mathrm{C} 2$ & $12 / 6 / 93$ & 1 & No & $3.83 \times 10^{13}$ & 42.5 & $2.24 \times 10^{3}$ & 0.62 & $2.21 \times 10^{46}$ & $3.56 \times 10^{38}$ \\
\hline $\mathrm{C} 2$ & $12 / 11 / 93$ & 1 & No & $1.37 \times 10^{14}$ & 5.9 & 9.94 & 1.61 & $4.39 \times 10^{48}$ & $3.56 \times 10^{38}$ \\
\hline $\mathrm{C} 2$ & $12 / 14 / 93$ & 1 & Yes & $2.30 \times 10^{14}$ & 2.0 & 1.18 & 2.89 & $2.10 \times 10^{49}$ & $3.56 \times 10^{38}$ \\
\hline $\mathrm{C} 1$ & $12 / 6 / 93$ & 6 & Yes & $1.33 \times 10^{14}$ & 1.5 & 1.23 & 3.19 & $5.01 \times 10^{47}$ & $3.68 \times 10^{37}$ \\
\hline $\mathrm{C} 2$ & $12 / 6 / 93$ & 6 & No & $3.14 \times 10^{13}$ & 18.0 & $1.89 \times 10^{3}$ & 0.92 & $4.32 \times 10^{44}$ & $4.05 \times 10^{37}$ \\
\hline $\mathrm{C} 2$ & $12 / 11 / 93$ & 6 & No & $1.10 \times 10^{14}$ & 2.5 & 6.43 & 2.50 & $1.05 \times 10^{47}$ & $4.05 \times 10^{37}$ \\
\hline $\mathrm{C} 2$ & $12 / 14 / 93$ & 6 & Yes & $1.91 \times 10^{14}$ & 0.9 & 1.11 & 4.31 & $4.10 \times 10^{47}$ & $4.05 \times 10^{37}$ \\
\hline
\end{tabular}




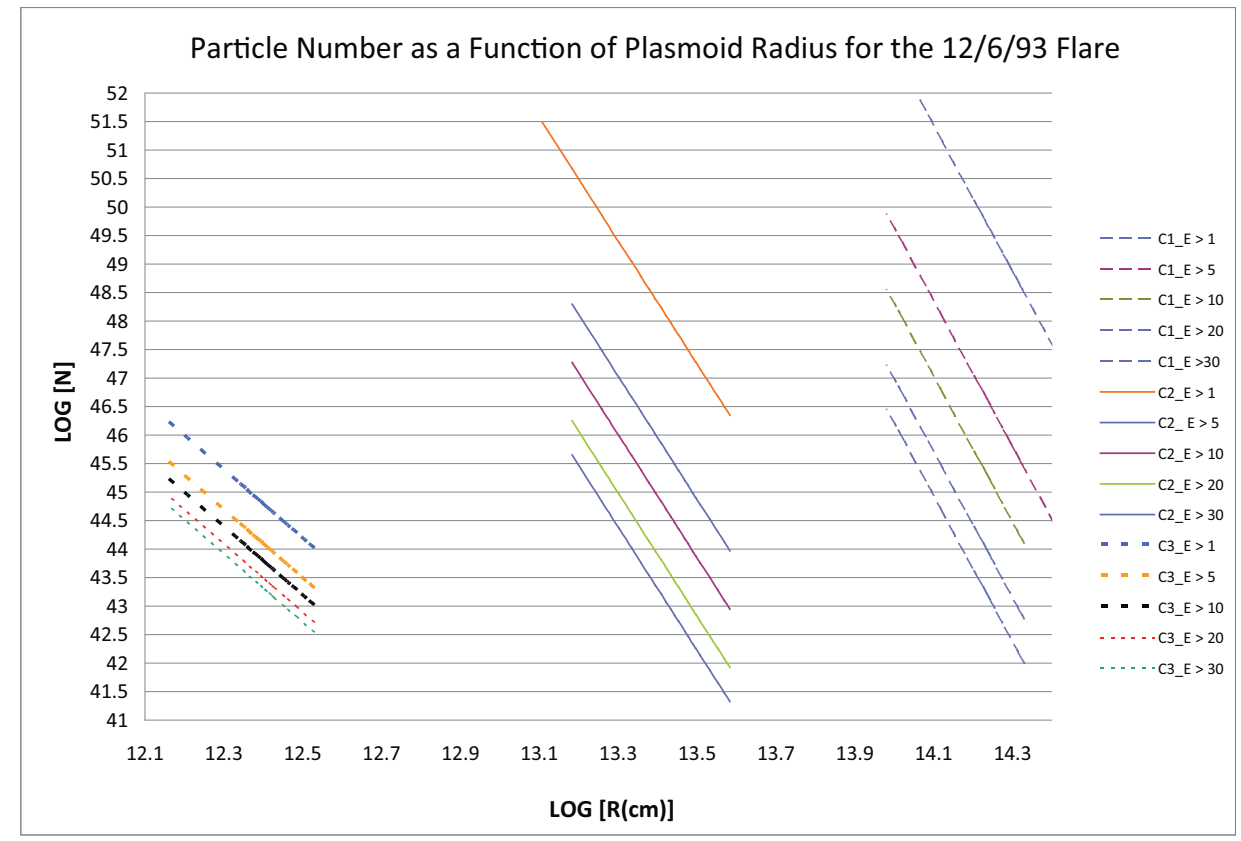

Fig. 6. - The total number of particles in the plasmoid, $N$, as a function of the radius of the model for each of the three components on December 6 . Notice that $N$ decreases with increasing $R$. The value of $N$ depends on $E_{\min }$. If the optically thin region of the spectrum is very steep then the dependence on $E_{\text {min }}$ is dramatic. This paper explores 5 models with various choices of $E_{\min }$ set at the values 1, 5,10, 20 and 30 . 


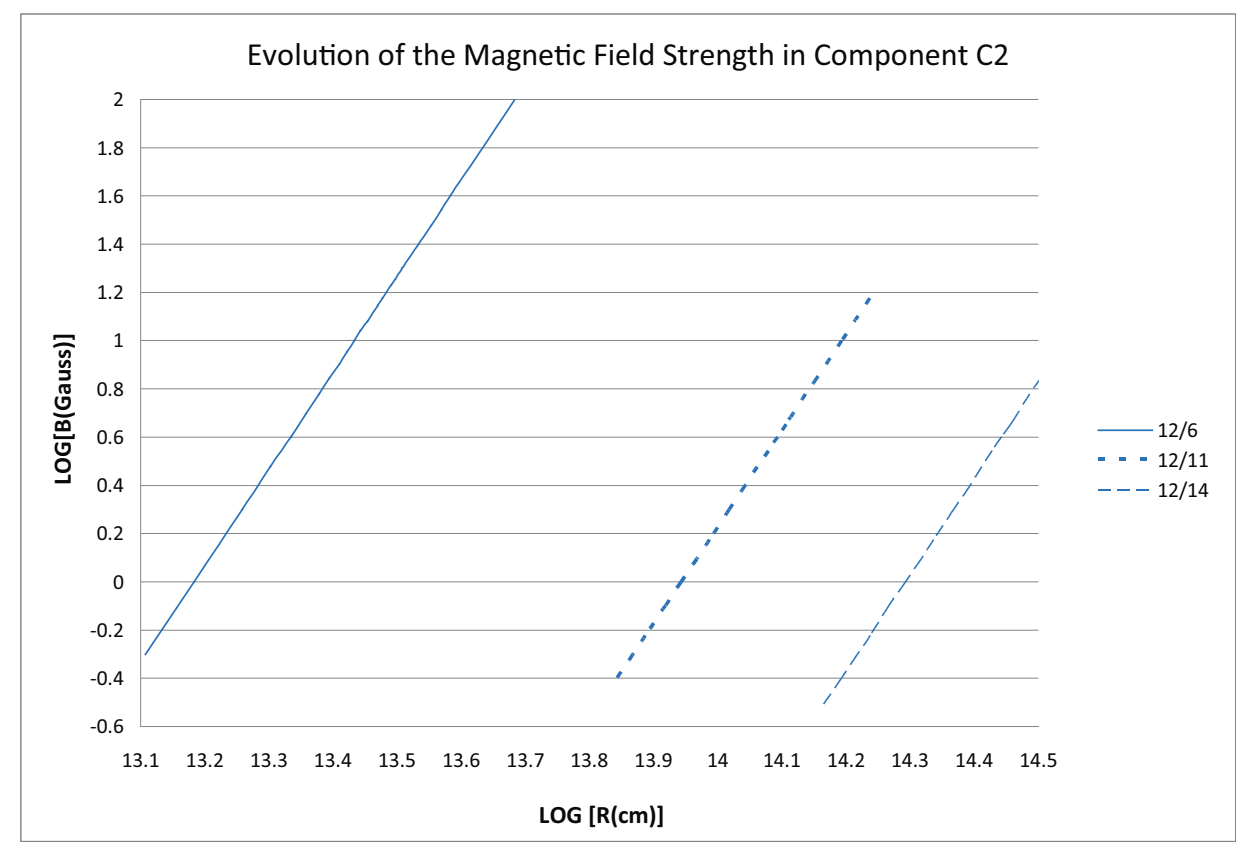

Fig. 7.- The magnetic field strength, $B$, as a function of the radius of the model for component $\mathrm{C} 2$ at three epochs, December 6, December 11 and December 14. Notice that $B$ increases with increasing $R$ at all epochs. 


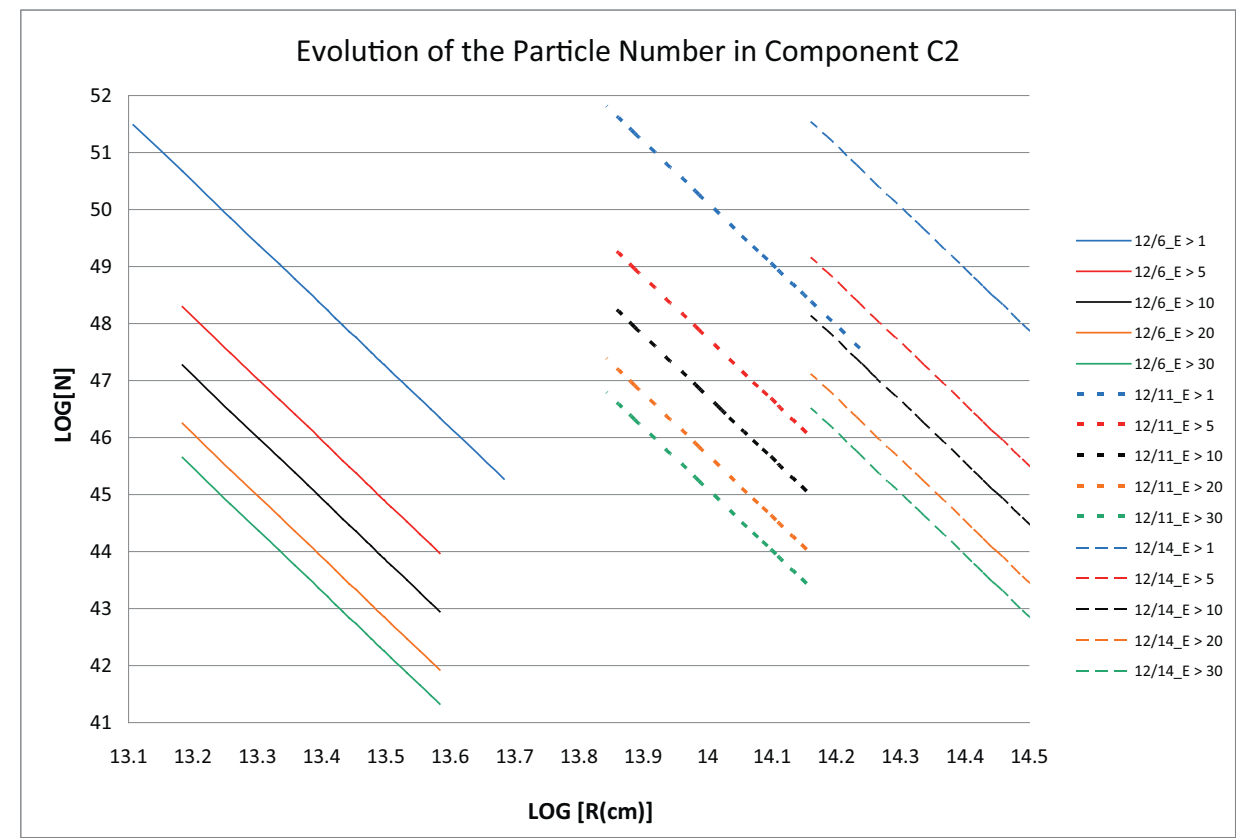

Fig. 8. - The total number of particles in the plasmoid, $N$, as a function of the radius of the model for for component C2 at three epochs, December 6, December 11 and December 14. Notice that $N$ decreases with increasing $R$ at all epochs. This paper models 5 choices of the parameter $E_{\min }: 1,5,10,20$ and 30. 


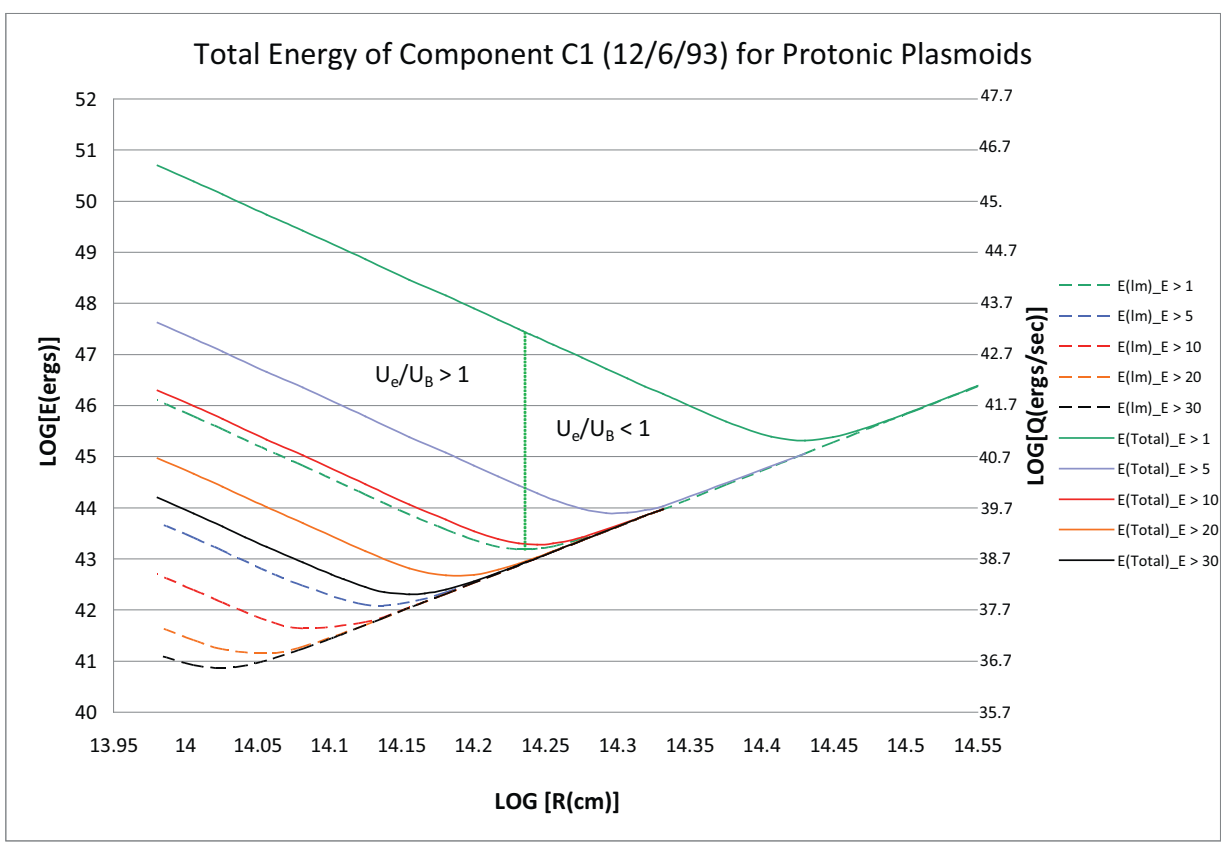

Fig. 9.- The left hand axis is the scale for the total energy stored, $E$ (Total), in C1 on December 6 for various protonic models of the plasmoid as a function of plasmoid radius, $R$. These values are contrasted with the lepto-magnetic energy, the energy stored in the leptons and the magnetic field, $E(\mathrm{~lm})$. The protonic energy is dominant except for large radii in which the magnetic field is large and particle number is small, see Figures 5 and 6. The vertical dotted green line connects the minimum energy lepto-magnetic solution for $E_{\min }=1$ (the dashed green curve) to the total energy of the protonic solution with $E_{\min }=1$ (the solid green curve). The value on the solid curve is much larger indicating that most of the energy is in the form of protonic kinetic energy for this "minimum energy solution." The right hand axis is the scale for the power required to energize and eject the plasmoid, $\mathrm{C} 1$, from the central engine as derived from the models of December 6. The total power, $Q$ (Total), is plotted assuming a protonic plasma. The power required to energize and eject just the lepto-magnetic component, $Q(\mathrm{~lm})$, is also plotted separately for comparison. The horizontal lines can be used to find the $Q$ (Total) that is associated with the minimum energy solutions (minimum $Q(\operatorname{lm})$ ) for each value of $E_{m i n}$. 


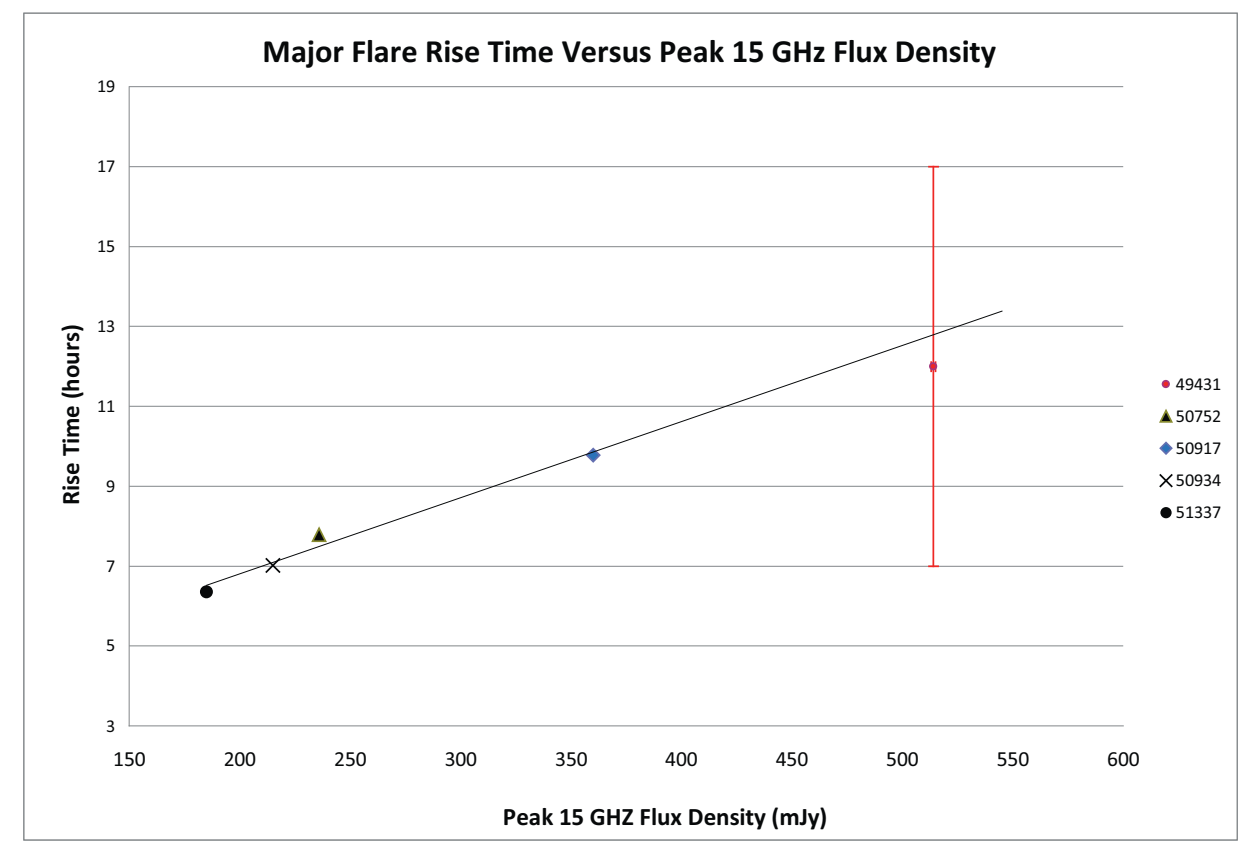

Fig. 10.- The rise time of 4 major flares as a function of peak $15 \mathrm{GHz}$ flux density from the long term $15 \mathrm{GHz}$ monitoring of Rushton et al (2010). The rise time of the flares at MJD 50917 and at MJD 50934 are derived from the dense data sampling at $8.4 \mathrm{GHz}$ and $2.3 \mathrm{GHz}$ in Dhawan et al (2000). The crude estimate of the rise time of the March 1994 flare from Rodriguez et al (1995) is also included. The data from the four well sampled flares is well fit by a line. 


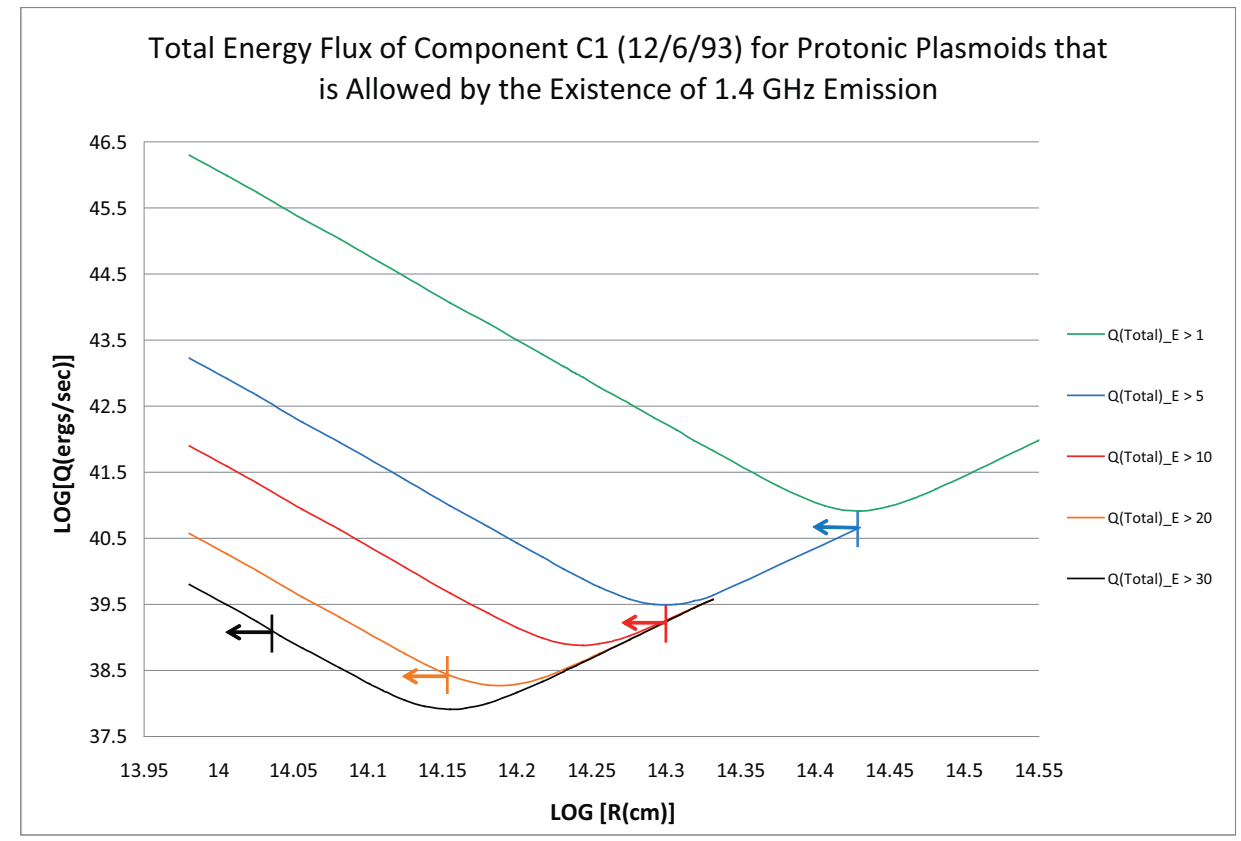

Fig. 11. - The same plots of $Q$ (Total) that appear in Figure 9 with the additional constraint that the energy of the particles responsible for the strong $1.4 \mathrm{GHz}$ emission satisfy, $E(1.4 \mathrm{GHz})>E_{\text {min }}$. The horizontal partitions separate the allowed regions of solution space from the forbidden regions of solution space for each value of $E_{\min }$. The arrows point toward the allowed region. 


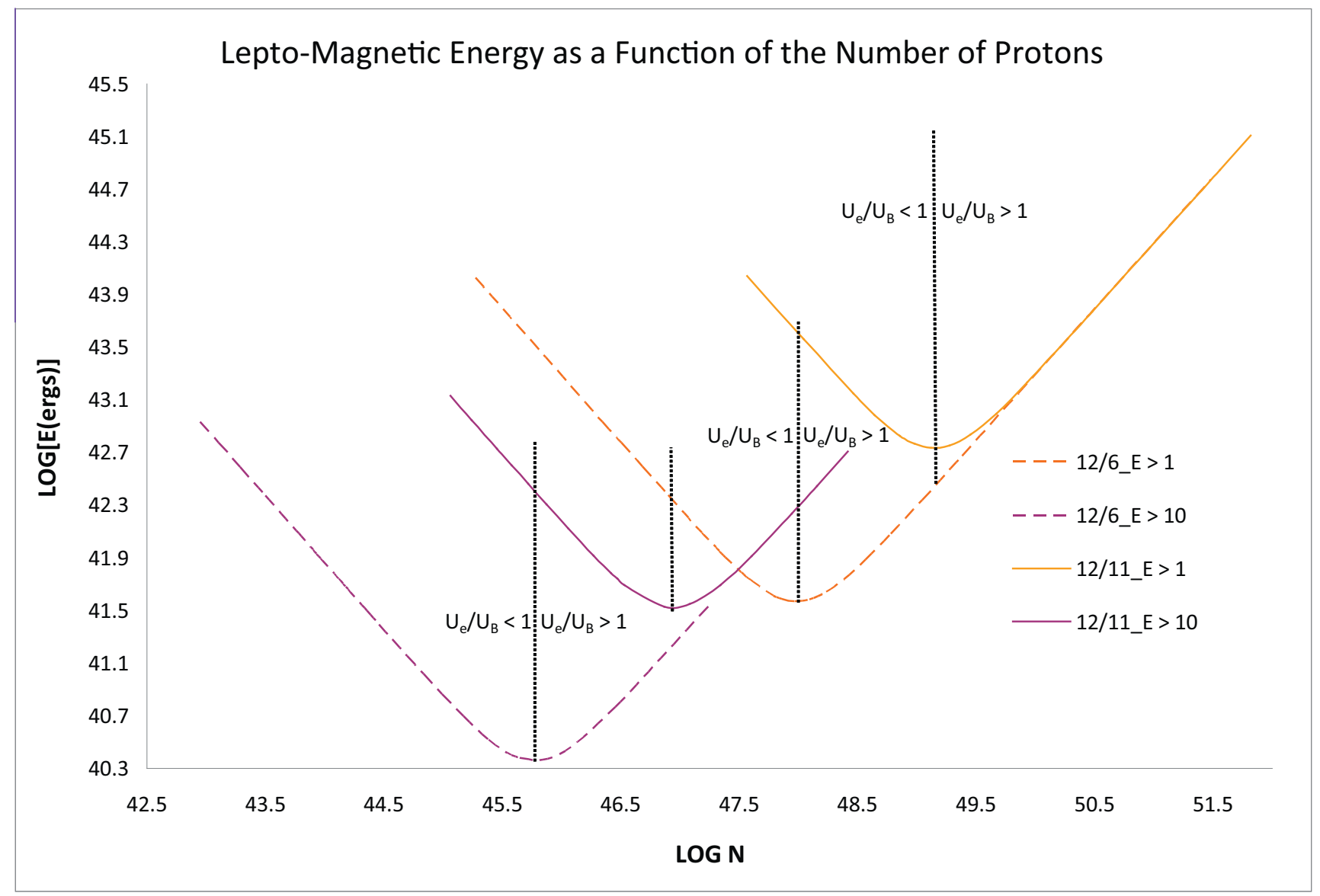

Fig. 12.- The lepto-magnetic energy, $E(\mathrm{~lm})$, in $\mathrm{C} 2$ on December 6 and December 11 as a function of the total number of particles, $N$, in the model of the plasmoid for $E_{\text {min }}=1$ and $E_{\text {min }}=5$. For protonic plasmoids, baryon number should be conserved. Thus, solutions at different epochs can be connected by a vertical line (i.e., the dashed black vertical lines). This figure shows that the protonic solutions require mechanical energy to be converted to magnetic energy as the plasmoids expand. 


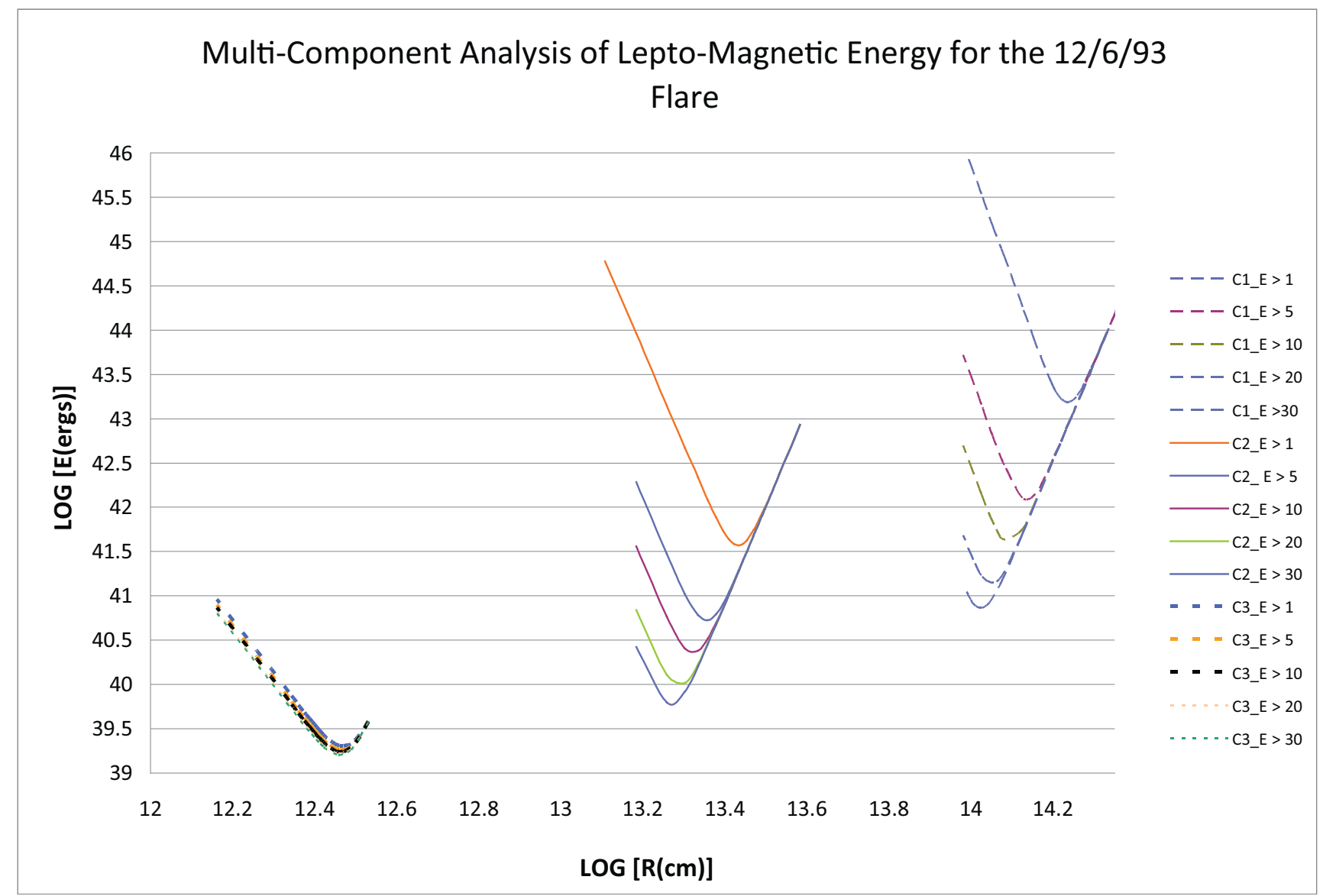

Fig. 13.- The lepto-magnetic energy, $E(\mathrm{~lm})$, in all three components on December 6 as a function of the spheroid radius, $R$, for the entire family of models. The lepto-magnetic energy in $\mathrm{C} 3$ is very insensitive to $E_{\min }$, so the curves almost overlap. It depends more on $E_{\max }$, the maximum lepton energy, because of the flat spectrum which was not that tightly constrained by the $234 \mathrm{GHz}$ measurement (it was 12 hours out of synchronization with the the bulk of the other data). A liberal value of $E_{\max }=10^{6}$ was chosen. In any event, the energy content is much smaller than for $\mathrm{C} 1$ and $\mathrm{C} 2$. 


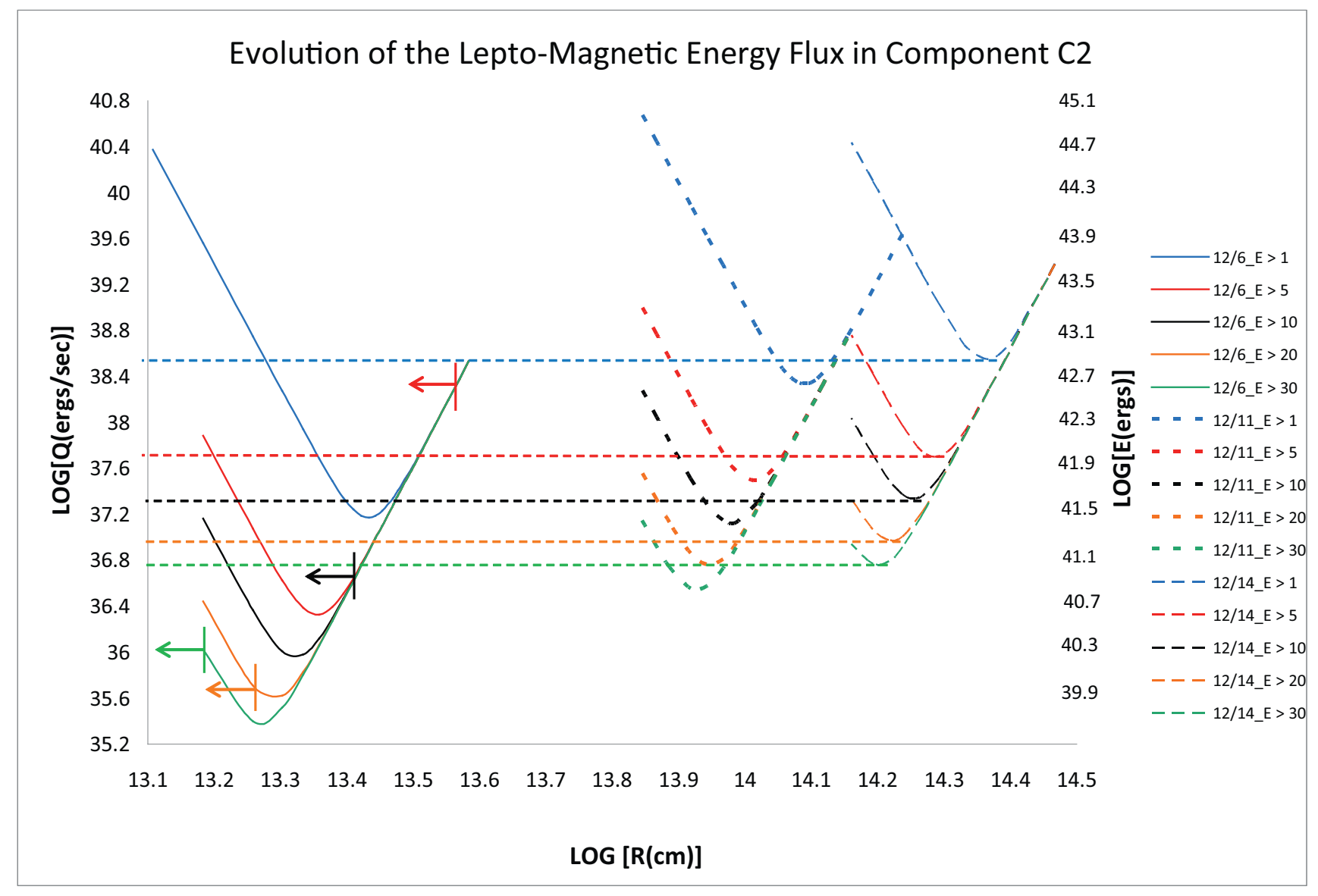

Fig. 14. - The scale on the right hand axis describes the time evolution in the lepto-magnetic energy, $E(\mathrm{~lm})$, in $\mathrm{C} 2$ from December 6 to December 14 as a function of the spheroid radius, $R$, for the entire family of plasmoid models. Color coded horizontal dashed lines connect color coded solutions with the same value of $E_{\min }$ and a conserved energy equal to the minimum energy on December 14. The scale on the left hand axis quantifies the lepto-magnetic energy flux, $Q(\mathrm{~lm})$, required to launch the leptonic plasma in $\mathrm{C} 2$ as deduced from the December 6 to December 14 models as a function of the spheroid radius, $R$, for the entire family of plasmoid models. In terms of energy flux the color coded horizontal dashed lines connect color coded solutions with the same value of $E_{\min }$ and a conserved energy flux equal to the minimum energy flux required to launch the plasmoid based on the fits of December 14 . This allows for an evolutionary track of magnetic energy conversion to mechanical energy as equipartition is approached at late times. The color coded vertical partitions separate the forbidden regions of solution space from the allowed regions of solution space (the allowed regions are indicated by the direction of the arrow). 


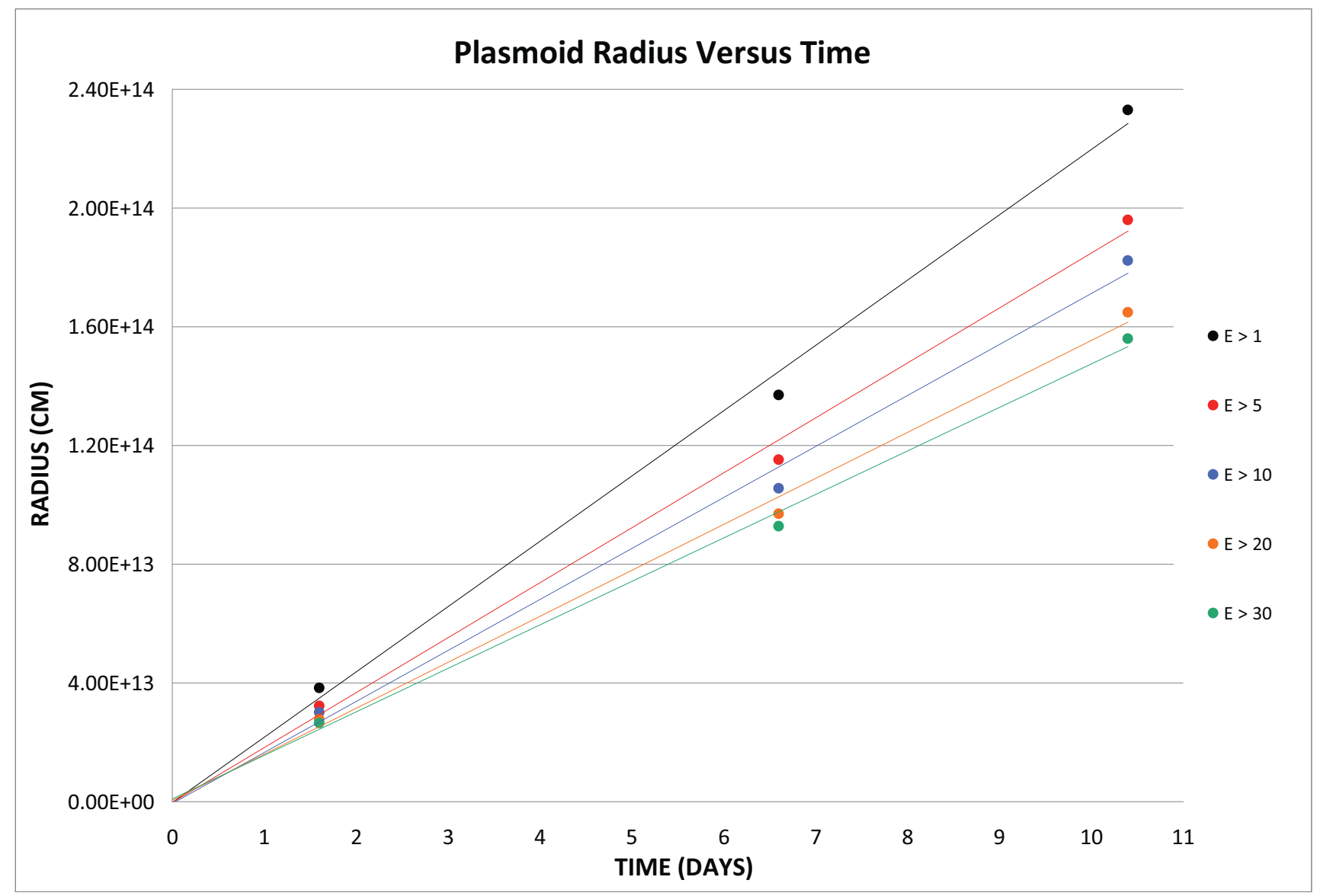

Fig. 15. - The radius of the family of models for $\mathrm{C} 2$ as a function of time. The zero point offset was adjusted so that the extrapolation of the fits to the data reach zero radius at zero time. 
brightness temperature is the equivalent blackbody temperature of the radiation assuming one is in the Planck regime, $h \nu \ll k_{b} T$. Consider a source in which the monochromatic intensity is $I(\nu)_{p}$ from a spheroid of radius $\mathrm{R}$. The brightness temperature associated with the spectral luminosity is

$$
\left(T_{b}\right)_{p}=\frac{S(\nu)_{o} \delta^{-1} c^{2}}{2 \Omega_{o} k_{b} \nu_{o}^{2}},
$$

where $\Omega_{o}$ and $\left(S_{\nu}\right)_{o}$, are the solid angle subtended by the source and the flux density, respectively. It was noted in Readhead (1994) that blazar jets tend to have $\left(T_{b}\right)_{p} \approx 10^{11}{ }^{\circ} \mathrm{K}$. Thus, Table 3 indicates that the values of $\left(T_{b}\right)_{p}$ are basically consistent with those expected in powerful astrophysical relativistic jets per the analysis of Readhead (1994). This value is below $\left(T_{b}\right)_{p} \approx 10^{12}{ }^{\circ} \mathrm{K}$ where the inverse Compton catastrophe occurs as a consequence of the inverse Compton radiation lifetime becoming much shorter than the synchrotron radiation lifetime (Kellermann \& Pauliny-Toth 1969). The ratio of inverse Compton luminosity, $L_{i c}$, to synchrotron Luminosity, $L_{\text {synch }}$ was calculated in Readhead (1994) as

$$
\begin{aligned}
& \frac{L_{i c}}{L_{\text {synch }}}=\left(\frac{T_{b} G(\alpha)^{1 / 5}}{10^{12.22}}\right)^{5}\left[1+\left(\frac{T_{b} G(\alpha)^{1 / 5}}{10^{12.22}}\right)^{5}\right], \\
& G(\alpha)= \\
& {\left[\frac{v_{\mathrm{op}} / \delta}{3.5 \mathrm{GHz}}+\frac{v_{\mathrm{op}} / \delta(1-\alpha)}{1 \mathrm{GHz}} \times\left[\left(\frac{v_{\text {high }}}{v_{\mathrm{op}}}\right)^{(1-\alpha)}-1\right]\right] f_{3}(\alpha)^{-4},}
\end{aligned}
$$

where $v_{\text {op }}$ is the peak in the observed SSA spectrum, $v_{\text {high }}$ is the highest frequency in the power law in the observers frame. This expression also requires the coefficient, $f_{3}(\alpha)$, that is given by equation (15c) of Scheuer and Williams (1968). In Table 4, the importance of the inverse Compton emission to the individual models is considered.

The values of $L_{i c} / L_{\text {synch }}$ in Table 4 represent a possible constraint on the solution spaces presented here. The ratios are not that sensitive to $v_{\text {high }}$ which is not determined by the

Table 4: Inverse Compton Losses in the Component Models

\begin{tabular}{ccccccccc}
\hline Component & Date & $\begin{array}{c}E_{\text {min }} \\
\end{array}$ & $\begin{array}{c}\text { Minimum } \\
\text { Energy }\end{array}$ & $\begin{array}{c}v_{\text {op }} \\
\mathrm{GHz}\end{array}$ & $\begin{array}{c}v_{\text {high }} \\
\text { GHz }\end{array}$ & $\alpha$ & $\begin{array}{c}T_{b} \\
10^{11}{ }^{\circ} \mathrm{K}\end{array}$ & $L_{\text {ic }} / L_{\text {synch }}$ \\
\hline $\mathrm{C} 1$ & $12 / 6 / 93$ & 1 & Yes & 2.9 & 50 & 2.2 & 1.91 & $3.23 \times 10^{-1}$ \\
$\mathrm{C} 2$ & $12 / 6 / 93$ & 1 & No & 9.5 & 50 & 1.7 & 0.62 & $1.40 \times 10^{-5}$ \\
$\mathrm{C} 2$ & $12 / 11 / 93$ & 1 & No & 5.5 & 50 & 1.7 & 1.61 & $1.06 \times 10^{-3}$ \\
$\mathrm{C} 2$ & $12 / 14 / 93$ & 1 & Yes & 2.6 & 50 & 1.7 & 2.89 & $1.01 \times 10^{-2}$ \\
$\mathrm{C} 1$ & $12 / 6 / 93$ & 6 & Yes & 2.9 & 50 & 2.2 & 3.19 & $1.45 \times 10^{1}$ \\
$\mathrm{C} 2$ & $12 / 6 / 93$ & 6 & No & 9.5 & 50 & 1.7 & 0.92 & $1.01 \times 10^{-4}$ \\
$\mathrm{C} 2$ & $12 / 11 / 93$ & 6 & No & 5.5 & 50 & 1.7 & 2.50 & $9.48 \times 10^{-3}$ \\
$\mathrm{C} 2$ & $12 / 14 / 93$ & 6 & Yes & 2.6 & 50 & 2.2 & 4.31 & $8.03 \times 10^{-2}$
\end{tabular}


available observations, so an arbitrary value of $50 \mathrm{GHz}$ is chosen. It was noted in Readhead (1994) that AGN tend to cluster at values of $T_{b}$ for which $L_{i c}$ starts to compete with $L_{\text {synch }}$, $\left(T_{b}\right)_{p} \gtrsim 10^{11}{ }^{\circ} \mathrm{K}$. This concept is consistent with the values in Table 4 . The one exception is $\mathrm{C} 1$, with the value of $E_{\text {min }}=6$, that appears to produce too much inverse Compton emission to be consistent with this concept. Thus, the results in Table 4 seem to favor the lower $E_{\min }$ range in the $\mathrm{C} 1$ solution space. Otherwise, it is concluded that the inverse Compton losses in Table 4 seem reasonable for a relativistic plasmoid.

According to Table 3, the maximum injected energy flux of component C1 is larger than that of component $\mathrm{C} 2$. Also, the larger minimum bound on the injection energy flux in Table 3 is for C2. Thus, the maximum injected power during the December 1993 flare is bounded to the range $4.05 \times 10^{37} \mathrm{erg} / \mathrm{sec}<Q$ (December, 1993) $\max <6.14 \times 10^{38} \mathrm{erg} / \mathrm{sec}$. Assume that there are oppositely directed plasmoids that are ejected simultaneously as commonly occurs in other observed flares. These components typically comprise only $\sim 10 \%$ of the total flux density due to the more intense redshifting when the ejections are directed away from the observer (Rodriguez and Mirabel 1999; Fender et al 1999; Miller-Jones et al 2005). It is noted that the estimates of $Q$ for the components $\mathrm{C} 1$ and $\mathrm{C} 2$ presented here are slightly high since this extra $10 \%$ of the flux appeared in their component flux densities. The existence of counter-ejecta would double the estimate above for the maximum total injected power from the central engine during the December 1993 flare, $9.1 \times 10^{37} \mathrm{erg} / \mathrm{sec}<$ $Q\left(\right.$ December 1993) $\max <1.22 \times 10^{39} \mathrm{erg} / \mathrm{sec}$. The central black hole in GRS $1915+105$ has a mass estimated at $14 M_{\odot}$ (Greiner et al 2001). Thus, the maximum impulsive power required to initiate the plasmoids is $0.05 L_{\mathrm{Edd}}<Q_{\max }<0.68 L_{\mathrm{Edd}}$ in terms of the Eddington luminosity, $L_{\text {Edd }}$.

\section{Discussion}

In this paper, a major flare from December 1993 from the micro-quasar GRS 1915+105 was analyzed in order to address four major unknowns from historical estimates of flare energy flux. These major uncertainties that were discussed in the Introduction are listed again here with the corresponding resolution implied by the detailed modeling described in the previous sections.

1. Is the plasma protonic? The answer seems to be no, it is electron-positron plasma. In section 4, it was shown that the energy fluxes required by the electron-proton plasma assumption exceed the radiated energy flux from the accretion flow (see Figure 11), implying that the plasmoids are driven by magnetic forces. Yet, the time evolution of C2 (see Figure 12) implies that mechanical energy is converted to magnetic energy 
over time. The time evolution history of this scenario is internally inconsistent. Thus, there is no causal time evolution if the protonic assumption is invoked.

2. What is the minimum electron energy, $E_{\min }$ ? In section 5 , it was shown that constraints imposed by the large magnetic fields in the compact plasmoids imply (through equation (16)) that small values of $E$ can produce the observed $1.4 \mathrm{GHz}$ flux densities. Since one must have $E>E_{\text {min }}$, large values of $E_{\text {min }}$ are forbidden. For the models of component, $\mathrm{C} 2,1<E_{\min }<6$.

3. There is uncertainty in the size of the region that produces the bulk of the radio emission. In section 5 , the models are used to show that the reason that interferometry does not resolve the preponderance of major flare flux in GRS 1915+105 is that the plasmoids responsible for this emission are $\sim 10 \mathrm{AU}$. The small plasmoid sizes are physically self-consistent as evidenced by the brightness temperatures of the plasmoids in Table 3. The volume used in previous estimates of the energy flux are typically two to three orders of magnitude too large.

\section{Is the minimum energy or the equipartition assumption justified? Definitely} not at early times, but possibly at late times. Figure 14 shows that there is no minimum energy evolutionary track through the solution space for C2 from December 6, 1993 to December 14, 1993.

The study presented here indicates that the major flares in GRS 1915+105 are discrete ejections of highly magnetized electron-positron plasma. The flares are associated with a strong long term magnetic transient that resides at the heart of the central engine that lasts $\sim 4.6 \times 10^{7}$ black hole spin periods (assuming a rapidly spinning black hole). As the flares propagate, they expand with a radial velocity $\approx 0.008 \mathrm{c}$ converting magnetic energy into a relativistically hot pair plasma. The models were able to bound the power required to energize and launch the most energetic flares, $8.10 \times 10^{37} \mathrm{erg} / \mathrm{sec}<Q$ (December 1993) < $1.22 \times 10^{39} \mathrm{erg} / \mathrm{sec}$. This result agrees very closely with the minimum energy estimate of Gliozzi et al (1999) for the March 1994 flare, if the radius of the emitting volume is corrected. They assumed a size of $R=7 \times 10^{15} \mathrm{~cm}$, which is a value much less than the interferometer beamwidth. This sub-beamwidth size was derived from a Gaussian fit algorithm to the size of the source (the unresolved core plus secondary) that was determined

in Rodriguez and Mirabel (1999). However, the model fits presented here indicate that the true size of the preponderance of the plasmoid emission should be much less than this. It is argued here that $R$ is likely to have been overestimated by a factor of 10 to 50 (based on the age of the flare when it was observed, $\sim 5$ days, and the results summarized in Table 3). The VLA at $8.3 \mathrm{GHz}$ cannot resolve a region of high emissivity $\sim 3 \times 10^{14} \mathrm{~cm}$ buried 
within a larger, inhomogeneous, low luminosity region of $\sim 7 \times 10^{15} \mathrm{~cm}$. From equation (5) of Gliozzi et al (1999), $Q \sim R^{0.5}$, thus the adjusted $Q$ values for an electron-positron plasma in Gliozzi et al (1999) are $3.0 \times 10^{38} \mathrm{erg} / \mathrm{sec}<Q\left(\right.$ March 1994) $<9.2 \times 10^{38} \mathrm{erg} / \mathrm{sec}$. They also considered magnetic dominance as a possible state of the ejected plasma, but did not pursue it in much detail. A second consistency check is provided by the strong flare observed with MERLIN on MJD 50752.1 in Fender et al (1999). They assumed a flare rise time of 12 hours and a plasmoid size of $10^{15} \mathrm{~cm}$ which is less than 0.14 of the MERLIN FWHM beamwidth. The plasmoid was unresolved in the image plane. Using Figure 10 to adjust the flare rise time to 8 hours and Table 3 to adjust the plasmoid radius to $\approx 2 \times 10^{14} \mathrm{~cm}$, equation (6) of Fender et al (1999) and Table 3 of Fender et al (1999) yield an "adjusted" minimum injection energy flux of $Q \approx 3.8 \times 10^{38} \mathrm{erg} / \mathrm{sec}$ for an electron-positron plasmoid, similar to the values presented here.

This study illustrates the investigative power provided by densely sampled broadband radio data in the study of major flares in GRS 1915+105. The power of this method is

a strong argument to initiate a study program that involves broadband quasi-simultaneous spectra (simultaneous to within a few hours and extending above $100 \mathrm{GHz}$ ) coordinated with high frequency VLBA observations $(15 \mathrm{GHz}$ and $22 \mathrm{GHz})$.

\section{REFERENCES}

Bicknell, G. 1994, ApJ 422542

deVries, W., Becker, R., White, R. 2006, AJ 131666

Dwahan, V., Mirabel, I.F., Rodriguez, L. 2000, ApJ 543373

Done, C., Wardzinski, G., Gierlinski, M. 2004, MNRAS 349393

Fender, R. et al, 1999, MNRAS 304865

Fender, R. and Pooley, 2000, MNRAS 318 L1

Fender, R., 2003, MNRAS 3401353

Foster, R. et al, 1996, ApJL 46781

Fuchs, Y. et al, 2003, A and A 409 L35

Harmon, B. et al, 1997, ApJL 47785

Ginzburg, V. and Syrovatskii, S. 1969, Annu. Rev. Astron. Astrophys. 7375 
Gliozzi,M. Bodo, G. and Ghisellini, G. 1999, MNRAS Letters 30340

Greiner, J., Cuby, J., McCaughrean, M. 2001, Nature 414522

Hawley, J., Krolik, K. 2006, ApJ 641103

Igumenshchev, I. V. 2008, ApJ 677317

Kantrowicz, A.R. and Petschek, H.E. 1966, In: Plasma Physics in Theory and Application. ed. by W.B. Kunkel (McGraw-Hill, New York) p. 148

Kellermann, K. I., \& Pauliny-Toth, I. I. K. 1969 ApJ, 155, L71

Kennel, C. and Coroniti, F. 1984 ApJ, 283, 649

Konigl, A. 1981, ApJ 243700

Krolik, J., Hawley, J., Hirose, S.2005, ApJ 622, 1008

Liang, E. and Li, H. 1995, A \& A 298 L45

Lind, K., Blandford, R. 1985, ApJ 295358

Marscher, a. et al 1979, ApJ 233498

Miller-Jones, J. et al 2005, MNRAS 363867

Mirabel, I.F., Rodriguez, L. 1994, Nature 37146

Mirabel, I.F. et al 1998, A \&A 330 L9

Moffet, A. 1975 in Stars and Stellar Systems, IX: Galaxies and the Universe, eds. A. Sandage, M. Sandage \& J. Kristan (Chicago University Press, Chicago), 211.

Pooley, G. and Fender, R. 1997, MNRAS 292925

Punsly, B. 2008, Black Hole Gravitohydromagnetics, second edition (Springer-Verlag, New York)

Readhead, A. 1994, ApJ 42651

Reynolds, C. S., Fabian, A., Celloti, A., Rees, M. 1996, MNRAS 283873

Reynolds, C., Punsly, B. Kharb, P., O’Dea, C. and Wrobel, J. 2009, 706851 
Ribo, M., Dhawan, V. Mirabel, F. 2004, Proc. of the 7th VLBI Network Symposium, Bachiller, R., Colomer, F., Desmurs, J., de Vicenete, P. (eds) Toledo, Spain astro-ph/0412657

Rodriguez, L., Gerard, E., Mirabel, I.F., Gomez, Y., Velaquez, A. 1995, ApJSS 101173

Rodriguez, L., Mirabel, I.F. 1999, ApJ 511398

Rushton, A., Spencer, E., Pooley, G. and Trushkin, S. 2010, MNRAS 4012611

Rushton, A., Spencer, E., Fender, R. and Pooley, G. 2011, A\& A accpeted http://xxx.lanl.gov/abs/1101.4945

Scheuer, P, Williams, P. 1968, Annu. Rev. Astron. Astrophys. 6321

Tucker, W. 1975, Radiation Processes in Astrophysics (MIT Press, Cambridge). 